\title{
RESTRICTION AND KAKEYA PHENOMENA FOR FINITE FIELDS
}

\author{
GERD MOCKENHAUPT AND TERENCE TAO
}

\begin{abstract}
The restriction and Kakeya problems in Euclidean space have received much attention in the last few decades, and are related to many problems in harmonic analysis, PDE, and number theory. In this paper we initiate the study of these problems on finite fields. In many cases the Euclidean arguments carry over easily to the finite setting (and are in fact somewhat cleaner), but there are some new phenomena in the finite case which deserve closer study.
\end{abstract}

In memory of Tom Wolff

\section{INTRODUCTION}

The purpose of this paper is to begin an investigation of restriction and Kakeya phenomena in the space $F^{n}$, where $F$ is a finte field of characteristic $\operatorname{char}(F)>2$. The corresponding phenomena in Euclidean space $\mathbf{R}^{n}$ have been intensively studied (see e.g. Tom Wolff's survey of the Kakeya phenomenon [49], and [35], [36], [8], [11] for a discussion of the restriction phenomenon) but it seems the finite field case has received far less attention. This is unfortunate, since the finite field case serves as a good model for the Euclidean case in that many of the technical difficulties (small angle issues, small separation issues, a logarithmically infinite number of scales, and Schwartz tails arising from the uncertainty principle) are eliminated. Also these finite field problems are closely related to other well-studied problems in number theory and arithmetic or geometric combinatorics, and techniques from these areas of mathematics may well be useful in attacking these problems. On the other hand, certain Euclidean space tools are not available in the finite field setting (e.g. Taylor series approximations, induction on scales, or combinatorial arguments based on the ordering of $\mathbf{R}$ ).

In this introduction we only give a brief overview of our results. Restriction estimates will be discussed in detail in Section 3 onwards, while Kakeya estimates will be discussed in detail in Section 8. Finally, we show a partial connection between the restriction and Kakeya problems in Section 9. Unfortunately the connection is not as tight as in the Euclidean case because one does not automatically have Taylor series approximations.

1991 Mathematics Subject Classification. 42B05, 11T24, 52C17. 
Restriction phenomena address the question of estimating the size of Fourier transforms of measures such as $f d \sigma$, where $d \sigma$ is surface measure on some set $S$ and $f$ is an arbitrary $L^{p}$ function. This problem in Euclidean space was first posed and partially solved by Stein in 1967 [18]. A typical inequality in Euclidean space is the Tomas-Stein inequality

$$
\left\|(f d \sigma)^{\vee}\right\|_{L^{2(n+1) /(n-1)}\left(\mathbf{R}^{n}\right)} \leq C_{n}\|f\|_{L^{2}\left(S^{n-1}\right)}
$$

where $f$ is an $L^{2}$ function on the sphere $S^{n-1}$ and $d \sigma$ is surface measure on the sphere.

In this paper we study this problem in the finite field setting for various algebraic varieties $S$; there are many sets $S$ of interest, but we shall mostly restrict our attention to cones and paraboloids. In two and three dimensions we shall be able to get reasonably good results, and in particular we can improve upon the standard Tomas-Stein restriction theorem for the paraboloid in three dimensions. Moreover, our results give reason to conjecture that in case -1 is not a square in $F$ the paraboliods are sharp $\Lambda(3)$-sets.

The Kakeya problem addresses the problem of the extent to which lines in different directions can overlap. (There are many interesting variants of this problem in which lines are replaced by other geometric objects, but we will not discuss them here). We will not prove too many new results for these problems, but we present some simplified proofs of various Euclidean arguments for the finite field setting, and show why this problem is connected to restriction problems and also to more classical problems in incidence geometry combinatorics.

This paper is partly of an expository nature; we have tried to make it accessible to readers who are not familiar with the restriction and Kakeya problems in the Euclidean case. We hope in particular that experts in combinatorics or number theory will be able to shed insight on these problems using techniques from those fields.

This work was conducted at UNSW and the University of Milano-Bicocca. The second author is a Clay Prize Fellow and is supported by the Packard Foundation.

\section{General notation}

If $A$ is a finite set, we use $|A|$ to denote the cardinality of $A$. We use $\delta_{i}$ to denote the Kronecker delta point mass at $i$, thus $\delta_{i}(j)=1$ if $i=j$ and $\delta_{i}(j)=0$ otherwise.

If $p$ is an exponent, we use $p^{\prime}$ to denote the dual exponent $1 / p+1 / p^{\prime}:=1$.

For positive numbers $\mathrm{x}$ and $\mathrm{y}$ we use $x \lesssim y$ or $x=O(y)$ to denote the estimate $x \leq C y$, where $C$ is a constant which depends on the dimension $n$ (and possibly on exponents such as $p, q$ ) but not on the underlying field $F$. We also use the notation $x \lesssim y$ to denote the estimate $x \leq C_{\varepsilon}|F|^{\varepsilon} y$ for all $\varepsilon>0$, where $C_{\varepsilon}$ is a constant which can depend on $\varepsilon$ but not on $F$. This notation is convenient for 
suppressing logarithmic powers of $F$. Unlike the Euclidean case, we do not have a logarithmically infinite number of scales, and so we expect that many of the logarithmic losses in this paper can in fact be removed. On the other hand, many of our estimates fail to be sharp by an actual power of $|F|$ rather than just an epsilon, and so the logarithmic losses are fairly insignificant by comparison in these cases.

We shall frequently use the following easy consequence of the Cauchy-Schwarz inequality: if $P, L$ are two finite sets with some relation $p \sim l$ between elements $p \in P, l \in L$, then

$$
\left|\left\{\left(p, l, l^{\prime}\right) \in P \times L \times L: p \sim l, p \sim l^{\prime}\right\}\right| \geq \frac{|\{(p, l) \in P \times L: p \sim l\}|^{2}}{|P|} .
$$

We shall be working in the vector space $F^{n}$, which we endow with counting measure $d x$, and the dual space $F_{*}^{n}$, which we endow with normalized counting measure $d \xi$ which assigns a mass of $|F|^{-n}$ to each point, so that $F_{*}^{n}$ has total mass 1 . Thus

$$
\int_{F^{n}} f(x) d x=\sum_{x \in F^{n}} f(x) \text { and } \int_{F_{*}^{n}} g(\xi) d \xi=\frac{1}{|F|^{n}} \sum_{\xi \in F_{*}^{n}} g(\xi) .
$$

The use of integrals for finite sums may appear a bit strange but this is a convenient normalization to prevent distracting powers of $|F|$ from appearing in the computations.

We fix $e: F \rightarrow S^{1}$ to be a non-principal character of $F$, i.e. a multiplicative function from $F$ to the unit circle which is not identically 1. For instance, if $F=\mathbf{Z} / p \mathbf{Z}$ for some prime $p$, one can take $e(x):=\exp (2 \pi i x / p)$. In practice the exact choice of this character will be irrelevant.

For any complex-valued function $f$ on $F^{n}$, we define the Fourier transform $\hat{f}$ on $F^{n}$ by

$$
\hat{f}(\xi):=\int_{F^{n}} f(x) e(-x \cdot \xi) d x
$$

where $x \cdot \xi$ is the inner product

$$
\left(x_{1}, \ldots, x_{n}\right) \cdot\left(\xi_{1}, \ldots \xi_{n}\right):=x_{1} \xi_{1}+\ldots+x_{n} \xi_{n} .
$$

The inverse of this transform is given by

$$
g^{\vee}(x):=\int_{F_{*}^{n}} g(\xi) e(x \cdot \xi) d \xi .
$$

We have the Parseval identity

$$
\int_{F^{n}} f_{1}(x) \overline{f_{2}(x)} d x=\int_{F_{*}^{n}} \hat{f}_{1}(\xi) \overline{\hat{f}_{2}(\xi)} d \xi
$$

the Plancherel identity

$$
\|f\|_{L^{2}\left(F^{n}, d x\right)}=\|\hat{f}\|_{L^{2}\left(F_{*}^{n}, d \xi\right)},
$$

and the intertwining of convolution and multiplication

$$
\hat{f}_{1} \hat{f}_{2}=\widehat{f_{1} * f_{2}} ; \quad \widehat{f_{1} f_{2}}=\hat{f}_{1} * \hat{f}_{2} .
$$


Note that $f_{1} * f_{2}$ is convolution using counting measure $d x$, while $\hat{f}_{1} * \hat{f}_{2}$ is convolution using normalized counting measure $d \xi$.

\section{THE RESTRICTION PROBLEM}

We begin by setting up some notation for the restriction problem.

Let $S \subset F_{*}^{n}$ be a non-empty set of frequencies, which in this paper will always be an algebraic variety in $F_{*}^{n}$. We endow $S$ with normalized "surface measure"

$$
\int_{S} g(\omega) d \sigma(\omega):=\frac{1}{|S|} \sum_{\xi \in S} g(\xi)
$$

thus $S$ has total mass 1 and by identifying measures with functions by dividing by the ambient measure we have

$$
(g d \sigma)^{\vee}(x)=\frac{1}{|S|} \sum_{\xi \in S} g(\xi) e(x \cdot \xi) .
$$

For any exponents $1 \leq p, q \leq \infty$, we let $R^{*}(p \rightarrow q)=R_{S}^{*}(p \rightarrow q)$ be the best constant such that the estimate

$$
\left\|(g d \sigma)^{\vee}\right\|_{L^{q}\left(F^{n}, d x\right)} \leq R^{*}(p \rightarrow q)\|g\|_{L^{p}(S, d \sigma)} .
$$

holds for all functions $g$ on $S$. Since $F$ is finite, the quantity $R^{*}(p \rightarrow q)$ will be a finite positive real. By duality, one can also define $R^{*}(p \rightarrow q)$ to be the best constant such that we have the restriction estimate

$$
\|\hat{f}\|_{L^{p^{\prime}}(S, d \sigma)} \leq R^{*}(p \rightarrow q)\|f\|_{L^{q^{\prime}}\left(F^{n}, d x\right)}
$$

for all functions $f$ on $F^{n}$.

For instance, it is immediate from the Plancherel's identity and Hölder's inequality that

$$
R^{*}(p \rightarrow \infty)=1 \text { for } 1 \leq p \leq \infty
$$

and

$$
R^{*}(p \rightarrow 2)=\left(\frac{|F|^{n}}{|S|}\right)^{1 / 2} \quad \text { for } 2 \leq p \leq \infty .
$$

For any algebraic variety $S$, i.e. a zero set in $F_{*}^{n}$ of a polynomial in $\mathbf{Z}\left[\xi_{1}, \ldots, \xi_{n}\right]$, the restriction problem for $S$ asks to determine the set of exponents $p$ and $q$ such that $R^{*}(p \rightarrow q) \leq C_{p, q}$, where $C_{p, q}$ does not depend on the underlying field $F$. One may think of the restriction problem as a quantitative way to understand the average size of exponential sums of the form

$$
\sum_{\xi \in S} g(\xi) e(x \cdot \xi)
$$

where $g$ is an arbitrary sequence in $l^{p}(S)$. To put it loosely, restriction problems are an attempt to understand exponential sums in which the coefficients $g(\xi)$ have no easily exploitable structure other than magnitude bounds. 
When the underlying field $F$ is replaced by $\mathbf{R}$, and $S$ is the unit sphere in $\mathbf{R}_{*}^{n}$, this problem was posed by E. M. Stein in 1967 [18], who observed (in our notation) that $R^{*}(p \rightarrow q)$ can be bounded for certain $q<\infty$ if $S$ has some non-trivial curvature.

To see how the Euclidean and the finite field restriction estimates are related consider the following heuristic argument (by standard arguments this can be made rigorous): Let $M \subset R^{n}$ be a compact $d$-dimensional smooth submanifold such that the translations of $M$ along normal vectors $n \neq 0$ at some point of $M$ do not intersect for $0<|n|<1 / R$ and suppose $\operatorname{supp}(f) \subset Q_{R}$, a cube of sidelength $\approx R$ around the origin. By the uncertainty principle $\hat{f}$ is roughly constant on a ball of radius $1 / R$. Hence, if $M_{R}$ is a $1 / R$-neighborhood of $M$ we have with

$$
\begin{aligned}
&\left.A=\left\{m \in Z^{n} \mid \operatorname{dist}\left(\frac{m}{R}, M\right) \leq \frac{1}{R}\right\} \quad \text { (note that }|A| \approx R^{d}\right), \\
& \int_{M_{R}}|\hat{f}|^{p^{\prime}} d x \approx \sum_{A} \int_{B\left(\frac{m}{R}, \frac{1}{R}\right)}|\hat{f}(x)|^{p^{\prime}} d x \\
& \approx \sum_{A} \operatorname{Vol}_{n}\left(B_{\frac{1}{R}}\left(\frac{m}{R}\right)\right)\left|\hat{f}\left(\frac{m}{R}\right)\right|^{p^{\prime}} \\
&=\frac{c_{n}}{R^{n}} \sum_{A}\left|\hat{f}\left(\frac{m}{R}\right)\right|^{p^{\prime}} .
\end{aligned}
$$

Let $f_{R}(x)=f(R x)$, thus $f_{R}$ has support in the unit cube and $\hat{f}\left(\frac{m}{R}\right)=R^{n} \hat{f}_{R}(m)$. Hence

$$
\int_{M_{R}}|\hat{f}|^{p^{\prime}} d x \approx \frac{R^{n p^{\prime}}}{R^{n}} \sum_{A}\left|\hat{f}_{R}(m)\right|^{p^{\prime}} .
$$

On the other hand we may assume $M_{R} \subset \bigcup_{|n R|<1}(n+M)$. Hence

$$
\int_{M_{R}}|\hat{f}|^{p^{\prime}} d x \lesssim \int_{n \in R^{n-d},|n|<\frac{1}{R}} \int_{n+M}|\hat{f}|^{p^{\prime}}
$$

By translation invariance and assuming a $\left(q^{\prime}, p^{\prime}\right)$-restriction inequality for $M$ :

$$
\begin{aligned}
\int_{M_{R}}|\hat{f}|^{p^{\prime}} d x & \lesssim \frac{1}{R^{n-d}} \sup _{n} \int_{n+M}|\hat{f}|^{p^{\prime}} d x \\
& \lesssim \frac{1}{R^{n-d}}\|f\|_{L^{q^{\prime}\left(Q_{R}\right)}}^{p^{\prime}}=\frac{R^{\frac{n p^{\prime}}{q^{\prime}}}}{R^{n-d}}\left(\int_{x \in Q_{1}}\left|f_{R}(x)\right|^{q^{\prime}} d x\right)^{p^{\prime} / q^{\prime}}
\end{aligned}
$$

With $g=f_{R}$ considered as a periodic function with Fourier coefficients $\hat{g}(m)$ we find:

i.e.

$$
\frac{R^{n p^{\prime}}}{R^{n}} \sum_{A}|\hat{g}(m)|^{p^{\prime}} \lesssim \frac{R^{\frac{n p^{\prime}}{q^{\prime}}}}{R^{n-d}}\left(\int_{x \in Q_{1}}|g(x)|^{q^{\prime}} d x\right)^{p^{\prime} / q^{\prime}}
$$

$$
\left(\sum_{A}|\hat{g}(m)|^{p^{\prime}}\right)^{\frac{1}{p^{\prime}}} \lesssim R^{\frac{d}{p^{\prime}}} R^{-\frac{n}{q}}\|g\|_{L^{q^{\prime}}\left(T^{n}\right)} .
$$

By duality we get

$$
\left(\int_{T^{n}}\left|\sum_{m \in A} a_{m} e^{i m \cdot x}\right|^{q}\right)^{\frac{1}{q}} \lesssim R^{\frac{d}{p^{\gamma}}} R^{-\frac{n}{q}}\left(\sum_{m \in A}\left|a_{m}\right|^{p}\right)^{\frac{1}{p}} .
$$


By eventually translating $A$ we may assume that $A \subset[0, R)^{n} \cap Z^{n}$. If we discretize the integral by a Riemann sum over cubes of sidelength $1 / R$ according to a result of Marcinkiewicz and Zygmund (see [50, Vol II p. 28]) the $L^{q}$-norm on the right hand side is equivalent to the discretized sum $(1<q<\infty)$ :

$$
\left(\frac{1}{R^{n}} \sum_{0 \leq x_{i}<R, x_{i} \in \mathbf{Z}}\left|\sum_{m \in A} a_{m} e^{i m \cdot \frac{x}{R}}\right|^{q}\right)^{\frac{1}{q}} \lesssim R^{-\frac{n}{q}} R^{d} R^{-\frac{d}{p}}\left(\sum_{m \in A}\left|a_{m}\right|^{p}\right)^{\frac{1}{p}} .
$$

Therefore, if $p \approx R$ is prime and $F=\mathbf{Z} / p \mathbf{Z}$ by identifying $A$ with a subset $S$ in $F_{*}^{n}$ we get, since $|S| \approx R^{d}$ :

$$
\left(\sum_{x \in F^{n}}\left|\frac{1}{|S|} \sum_{m \in S} a_{m} e(m \cdot x)\right|^{q}\right)^{\frac{1}{q}} \lesssim\left(\frac{1}{|S|} \sum_{m \in S}\left|a_{m}\right|^{p}\right)^{\frac{1}{p}}
$$

i.e.

$$
R_{S}^{*}(p \rightarrow q) \lesssim 1
$$

Note that $S \subset F_{*}^{n}$ depends on the prime $p$, i.e. here $S$ is not given by a fixed algebraic equation over $\mathbf{Z}$.

A large amount of work, especially in the three-dimensional case, has since been done on the restriction problems (see e.g. [36], [8], [48], [43] and the references therein); notably for the case of a circle in $\mathbf{R}^{2}$ the problem was solved by C. Fefferman and E. M. Stein in [18]. The restriction problem is also connected to problems in dispersive and wave equations, and to certain problems in number theory; we refer the reader to [11], [49], [29] for further discussion of these connections.

In this paper we shall mostly restrict ourselves to the case when $S$ is a hypersurface, however the lower codimension case is also extremely interesting (see [14], [29]). In fact, we shall mostly concern ourselves with paraboloids and cones in $F_{*}^{n}$; we have avoided the sphere as the Fourier transform of surface measure is not as easy to compute in the finite field case. Other quadratic surfaces are also of interest, see e.g. [37].

\section{General Restriction theory}

In this section we collect some general (and rather easy) facts about the quantity $R^{*}(p \rightarrow q)$. We shall assume that $|S| \sim|F|^{d}$ for some $0<d<n$ (this is the finite field analogue of $S$ having dimension $d$ ).

From Hölder's inequality and the embedding $l^{p_{1}} \subset l^{p_{2}}$ for $p_{1} \geq p_{2}$ we see that

$$
R^{*}\left(p_{1} \rightarrow q\right) \leq R^{*}\left(p_{2} \rightarrow q\right) \text { when } p_{1} \geq p_{2}
$$

and

$$
R^{*}\left(p \rightarrow q_{1}\right) \leq R^{*}\left(p \rightarrow q_{2}\right) \leq|F|^{n\left(\frac{1}{q_{2}}-\frac{1}{q_{1}}\right)} R^{*}\left(p \rightarrow q_{1}\right) \text { when } q_{1} \geq q_{2} .
$$

From these inequalities and (5) we see that

$$
R^{*}(p \rightarrow q) \geq|F|^{-n\left(\frac{1}{2}-\frac{1}{q}\right)+} R^{*}(\infty \rightarrow 2)=|F|^{-n\left(\frac{1}{2}-\frac{1}{q}\right)+}\left(\frac{|F|^{n}}{|S|}\right)^{1 / 2} .
$$


Here we use $a_{+}$as shorthand for $\max (a, 0)$. Since $|S| \sim|F|^{d}$, we thus see that $R^{*}(p \rightarrow q)$ can only be bounded by $O(1)$ if

$$
q \geq \frac{2 n}{d}
$$

From (8) we have in particular that

$$
R^{*}(2 \rightarrow q) \gtrsim|F|^{n / q}|S|^{-1 / 2}
$$

whenever $2 \leq q \leq \infty$. If the $\gtrsim$ can be replaced by $\sim$, then $R^{*}(2 \rightarrow q)$ resembles the $\Lambda(q)$-constant of the set $S$ (see e.g. [5]); roughly speaking, this means that the exponentials $\{e(x \cdot \xi): \xi \in S\}$ form an essentially orthogonal set in $L^{q}$.

Also, if we test (2) with $g$ equal to a Dirac delta $g=\delta_{\eta}$ for some $\eta \in S$, we obtain

$$
|S|^{-1}|F|^{n / q} \leq R^{*}(p \rightarrow q)|S|^{-1 / p}
$$

which implies that $R^{*}(p \rightarrow q)$ can only be bounded by $O(1)$ if

$$
q \geq \frac{n p^{\prime}}{d} \text {. }
$$

As we shall see, in some cases the necessary conditions (9), (10) for the boundedness of $R^{*}(p \rightarrow q)$ are also sufficient. However, if $S$ is somehow "flat", then one can improve the above necessary conditions.

For instance, suppose $S$ contains an affine subspace $V \subset F_{*}^{n}$ of dimension $k$. Then if we test (2) with $g$ equal to the characteristic function $\chi_{V}$, we obtain

$$
|F|^{k}|S|^{-1}|F|^{(n-k) / q} \leq R^{*}(p \rightarrow q)|F|^{k / p}|S|^{-1 / p}
$$

which yields the condition

$$
q \geq p^{\prime} \frac{n-k}{d-k}
$$

The problem of estimating $R^{*}(p \rightarrow q)$, in particular estimates for $R^{*}(\infty \rightarrow q)$, is related to the Hardy-Littlewood majorant problem, see $[21,26]$. This problem asks whether for any $2 \leq p<\infty$ and any functions $f, g$ with $|\widehat{f}(\xi)| \leq \widehat{g}(\xi)$ for all $\xi \in F_{*}^{n}$, one has $\|f\|_{p} \leq C_{p}(F)\|g\|_{p}$ with $C_{p}(F)$ independent of $F$. It was observed by Hardy and Littlewood in [21] that this is true with $C_{p}(F)=1$ for even integer $p$, but examples of Boas, Bachelis, and Fournier show that $C_{p}(F)$ grows unboundedly with $F$ for all other values of $p$ (see e.g. [19]). A quantitative lower bound for the grows of $C_{p}(F)$ is given in [29], e.g. for $2<p<4$ one has $C(F) \geq|F|^{\frac{c_{p}}{\log \log |F|}}$ with $c_{p}>0$ independent of $F$. However, this failure is only $O\left(|F|^{\varepsilon}\right)$ for any $\varepsilon>0$, so it is still possible that a weaker version $\|f\|_{p} \lesssim\|g\|_{p}$ holds. If this were true then we would have in particular that (for all $S$ )

$$
\mathbf{R}^{*}(\infty \rightarrow q) \approx\left\|(d \sigma)^{\vee}\right\|_{q}
$$

For further discussion see [29]. 


\section{EVEn EXPONENTS}

When $q$ is an even integer one can compute $R^{*}(p \rightarrow q)$ quite easily just from Plancherel's theorem. For instance:

Lemma 5.1. Let $q=2 k$ be a positive even integer, and suppose that for any $\eta \in F_{*}^{n}$ the number of solutions to the equation

$$
\eta=\xi_{1}+\ldots+\xi_{k} ; \quad \xi_{1}, \ldots, \xi_{k} \in S
$$

is bounded by $A$. Then we have

$$
R^{*}(2 \rightarrow 2 k) \leq A^{1 / 2 k}|F|^{n / 2 k}|S|^{-1 / 2} .
$$

Proof We have to show that

$$
\|\widehat{f d \sigma}\|_{L^{2 k}\left(F^{n}, d x\right)} \leq A^{1 / 2 k}|F|^{n / 2 k}|S|^{-1 / 2}\|f\|_{L^{2}(S, d \sigma)} .
$$

Raising this to the $k^{\text {th }}$ power and applying Plancherel, this becomes

$$
\|f d \sigma * \ldots * f d \sigma\|_{L^{2}\left(F_{*}^{n}, d \xi\right)} \leq A^{1 / 2}|F|^{n / 2}|S|^{-k / 2}\|f\|_{L^{2}(S, d \sigma)}^{k}
$$

where the convolution contains $k$ copies of $f d \sigma$.

It is now clear that we can assume $f$ is positive. By hypothesis we have

$$
\|d \sigma * \ldots * d \sigma\|_{L^{\infty}\left(F_{*}^{n}, d \xi\right)} \leq A|F|^{n}|S|^{-k}
$$

while from Fubini's theorem we have

$$
\left\|f^{2} d \sigma * \ldots * f^{2} d \sigma\right\|_{L^{1}\left(F_{*}^{n}, d \xi\right)}=\|f\|_{L^{2}(S, d \sigma)}^{2 k} .
$$

The claim then follows by taking the geometric mean of these estimates and applying Cauchy-Schwartz.

The above lemma has seen many applications, e.g. Sidon employed it in his study of lacunary Fourier series. A variant in Euclidean spaces using Hausdorff-Young's inequality instead of Plancherel's identity is the crucial tool in settling the restriction problem for circles and more general curves in $\mathbf{R}^{2}$ (see e.g. [18], [34]). The continuous variants as well as related estimates in the theory of bilinear $X^{s, b}$-estimates are also useful in non-linear PDE's (see e.g. [7], [27], [40], etc.).

As an application we consider the polynomial curve

$$
S:=\gamma(F) \text {, }
$$

where

$$
\gamma(t):=\left(t, t^{2}, \ldots, t^{n}\right)
$$

The $n$ coordinates of $\gamma\left(t_{1}\right)+\ldots+\gamma\left(t_{n}\right)$ are just the first $n$ symmetric functions of $t_{1}, \ldots, t_{n}$. If $\operatorname{char}(F)>n$ Newton's formulae show that the $t_{1}, \ldots, t_{n}$ are determined up to permutations. Thus we can apply the above Lemma with $k:=n$ and $A:=n$ ! to conclude that $R^{*}(2 \rightarrow 2 n)$ is bounded. From $(6),(7)$ we thus see that $R^{*}(p \rightarrow q)$ is bounded whenever

$$
q \geq 2 n, n p^{\prime}
$$


This exactly matches the necessary conditions (9), (10) (with $d=1$ ). Note that if $F$ has characteristic $n$ or less, then $\gamma$ becomes degenerate and $S$ is contained inside a proper subspace of $F_{*}^{n}$.

For the rest of the section we consider the paraboloid

$$
S:=\left\{(\xi, \xi \cdot \xi): \xi \in F_{*}^{n-1}\right\} .
$$

We apply Lemma 5.1 with $k:=2$. To find $A$, we need to bound solutions to the problem

$$
\xi_{1}+\xi_{2}=\eta^{\prime}, \quad \xi_{1} \cdot \xi_{1}+\xi_{2} \cdot \xi_{2}=\tau
$$

for any $\eta^{\prime} \in F^{n-1}$ and $\tau \in F$. If one fixes $n-2$ of the coordinates of $\xi_{1}$, then the remaining coordinate obeys a quadratic equation (after substituting $\xi_{2}=\eta^{\prime}-\xi_{1}$ ) and thus has at most two solutions. Thus we may take $A=2|F|^{n-2}$. From Lemma 5.1 we thus see that $R^{*}(2 \rightarrow 4)$ is bounded. (The same argument gives $R^{*}(2 \rightarrow 2 k$ ) for any even integer $2 k$, but this is in any event implied from the $2 k=4$ case by $(7))$.

From (9), (10) with $d:=n-1$ we obtain the necessary conditions

$$
q \geq \frac{2 n}{n-1}, \frac{n p^{\prime}}{n-1}
$$

for the boundedness of $R^{*}(p \rightarrow q)$. If $n=2$ then the above discussion (combined with (6), (7)) shows that these necessary conditions are in fact sufficient; this was first observed by Zygmund.

For $n>2$ it is possible for the paraboloid to contain lines (since $\xi \cdot \xi$ can vanish even when $\xi \neq 0$ ) or even higher-dimensional subspaces, at which point one can improve the above necessary conditions. To avoid these complications let us restrict ourselves to the case $n=3$, with -1 not a square number in $F$ (so that $|F|$ is a power of a prime $p=3(\bmod 4))$. In this case the parabola does not contain any lines, and the necessary conditions become

$$
q \geq 3, \frac{3 p^{\prime}}{2} .
$$

It seems reasonable to conjecture that the above conditions are still sufficient. In other words, we conjecture that $R^{*}(2 \rightarrow 3)$ is bounded - which is substantially stronger than the bound of $R^{*}(2 \rightarrow 4)$ obtained earlier. We remark that the corresponding conjecture for the paraboloid in $\mathbf{R}^{3}$ is a challenging open question; see e.g. [44] for some recent progress.

As partial evidence of this conjecture we present

Proposition 5.2. If $n=3,-1$ is not a square number, and $S$ is the paraboloid (13), then we have

$$
R^{*}(8 / 5 \rightarrow 4) \lesssim 1
$$

Note that this is on the line $q=3 p^{\prime} / 2$. This only improves the " $p$ " index of the $R^{*}(2 \rightarrow 4)$ result, but we will give an improvement to the " $q$ " index later in 
Theorem 6.2. The logarithmic factor can probably be removed, but we will not do so here.

Before we prove Proposition 5.2 we need the following well-known incidence geometry lemma (see e.g. [4]).

Proposition 5.3. Let $P$ be a collection of points in $F^{2}$, and let $L$ be a collection of lines in $F^{2}$. Then

$$
|\{(p, l) \in P \times L: p \in l\}| \leq \min \left(|P|^{1 / 2}|L|+|P|,|P||L|^{1 / 2}+|L|\right)
$$

Proof By the duality of points and lines in $F^{2}$ (or more precisely, projective space $P F^{2}$ ) it suffices to verify the first bound. Denote the set on the left-hand side by I. By (1) we have

$$
\left|\left\{\left(p, l, l^{\prime}\right) \in P \times L \times L: p \in l ; p \in l^{\prime}\right\}\right| \geq \frac{|I|^{2}}{|P|} .
$$

Fix $l, l^{\prime}$. If $l \neq l^{\prime}$ then there is at most one point $p$ which contributes to the left-hand side. Thus

$$
|I|+|L|^{2}-|L| \geq \frac{|I|^{2}}{|P|}
$$

which we re-arrange as

$$
(|I|-|P| / 2)^{2} \leq|P||L|^{2}-|P||L|+|P|^{2} / 4 \leq\left(|P|^{1 / 2}|L|+|P| / 2\right)^{2} .
$$

The claim follows.

We can now prove Proposition 5.2.

Proof This will be analogous to the " $12 / 7$ " estimate in [32], [42]. We want to show that

$$
\left\|(f d \sigma)^{\vee}\right\|_{L^{4}\left(F_{*}^{n}, d \xi\right)} \lesssim\|f\|_{L^{8 / 5}(S, d \sigma)} \cdot
$$

Since we are allowing ourselves to lose logarithmic powers of $|F|$ we may restrict ourselves to characteristic functions $f=\chi_{E}$ by the usual dyadic pigeonholing argument, where $E$ is any non-empty subset of $S$.

Fix E. By arguing as in Lemma 5.1, it suffices to show that

$$
\left\|\chi_{E} d \sigma * \chi_{E} d \sigma\right\|_{L^{2}\left(F_{*}^{n}, d \xi\right)} \lesssim\left\|\chi_{E}\right\|_{L^{8 / 5}(S, d \sigma)}^{2} .
$$

Expanding this all out, this becomes

$$
\sum_{\omega_{1}, \omega_{2}, \omega_{3}, \omega_{4} \in E: \omega_{1}+\omega_{2}=\omega_{3}+\omega_{4}} 1 \lesssim|E|^{5 / 2} .
$$

Since $E \subset S$, it will suffice to show that

$$
\sum_{\omega_{2}, \omega_{3} \in E: \omega_{3}-\omega_{2} \in S-\omega_{1}} 1 \lesssim|E|^{3 / 2}
$$

for all $\omega_{1} \in E$. Since $S$ is invariant under Galilean transformations

$$
g_{a}: F_{*}^{n-1} \times F_{*} \ni(\xi, \tau) \mapsto(\xi+a, \tau+2 \xi \cdot a+a \cdot a)
$$


the above sum transforms under $g_{-\eta_{1}}$, where $\left(\eta_{1}, \eta_{1} \cdot \eta_{1}\right)=\omega_{1}$, into

$$
\sum_{\omega_{2}, \omega_{3} \in E^{\prime}: \omega_{3}-\omega_{2} \in S} 1
$$

with $E^{\prime}=g_{-\eta_{1}}(E)$. We may take $\omega_{2}, \omega_{3} \neq 0$ since the contribution of $\omega_{2}=0$ or $\omega_{3}=0$ is $O(|E|)$. If we write $E^{\prime} \backslash\{0\}=\{(\xi, \xi \cdot \xi): \xi \in P\}$ for some $P \subset F_{*}^{2}$, and change coordinates such that $\omega_{2}=(\xi, \xi \cdot \xi)$ and $\omega_{3}=(\eta, \eta \cdot \eta)$, the condition $\omega_{3}-\omega_{2} \in S$ then becomes $\xi \cdot \eta=\xi \cdot \xi$, and we reduce to showing

$$
|\{(\xi, \eta) \in P \times P: \xi \cdot \eta=\xi \cdot \xi\}| \lesssim|P|^{3 / 2}
$$

For any $\xi \in P$, let $l(\xi)$ denote the line

$$
l(\xi):=\left\{\eta \in F_{*}^{2}: \xi \cdot \eta=\xi \cdot \xi\right\}
$$

and let $L$ denote the set of all such lines $l(\xi)$. Since -1 is not a square we see that these lines are all distinct, so $|L|=|P|$. Our task is then to show the incidence bound

$$
|\{(p, l) \in P \times L: p \in l\}| \lesssim|P|^{3 / 2} .
$$

But this follows from Proposition 5.3.

\section{Tomas-Stein type ARguments}

Let $S$ be any subset of $F_{*}^{n}$. Suppose we wish to estimate the quantity $R^{*}(2 \rightarrow q)$ for some $q$. If we square (3) and apply Plancherel, we see that

$$
\left|\left\langle f, f *(d \sigma)^{\vee}\right\rangle\right| \leq R^{*}(2 \rightarrow q)^{2}\|f\|_{L^{q^{\prime}\left(F^{n}, d x\right)}}\|f\|_{L^{q^{\prime}\left(F^{n}, d x\right)}}
$$

and furthermore that $R^{*}(2 \rightarrow q)$ is the best constant with this property.

It is thus of interest to study the object $(d \sigma)^{\vee}$. At the origin we clearly have $(d \sigma)^{\vee}(0)=1$, however away from the origin we often have some decay. To this end we introduce the Bochner-Riesz kernel

$$
K(x):=(d \sigma)^{\vee}(x)-\delta_{0}(x)
$$

which is just $(d \sigma)^{\vee}$ with the origin removed. This kernel is the finite field analogue of (a dyadically localized portion of) the kernel for Bochner-Riesz means in $\mathbf{R}^{n}$, see e.g. [18], [13] for further discussion.

Suppose that we have the decay estimate

$$
\|K\|_{L^{\infty}\left(F^{n}, d x\right)} \lesssim|F|^{-\tilde{d} / 2}
$$

for some exponent $0<\tilde{d}<n$; this quantity is sometimes referred to as the Fourier dimension of $S$.

If $|S| \sim|F|^{d}$ for some $0<d<n$, then from Plancherel we have

$$
\left\|(d \sigma)^{\vee}\right\|_{L^{2}\left(F^{n}, d x\right)}=\|d \sigma\|_{L^{2}\left(F_{*}^{n}, d \xi\right)}=|F|^{(n-d) / 2},
$$

which implies that $\tilde{d} \leq d$. In some cases (e.g. the parabola) the two notions of dimension are equal, but when $S$ has some arithmetic closure properties the Fourier dimension is often smaller than the ordinary dimension. 
If we could somehow ignore the $x=0$ component of $(d \sigma)^{\vee}(x)$, then from (15), then we would have

$$
\left|\left\langle f, f *(d \sigma)^{\vee}\right\rangle\right| \lesssim|F|^{-\tilde{d} / 2}\|f\|_{L^{1}\left(F^{n}, d x\right)}\|f\|_{L^{1}\left(F^{n}, d x\right)}
$$

which would then imply $R^{*}(2 \rightarrow \infty) \lesssim|F|^{-\tilde{d} / 4}$. Of course this is absurd by (4), however for interpolation purposes this is morally true ${ }^{1}$. A precise statement is the following (which is closely related to the formulation of the Tomas-Stein argument given in Bourgain [6]):

Lemma 6.1 (Tomas-Stein argument). Let $p, q \geq 2$, and suppose that (15) holds. Then for any $0<\theta<1$ we have

$$
R^{*}(p \rightarrow q / \theta) \lesssim 1+R(p \rightarrow q)^{\theta}|F|^{-\tilde{d}(1-\theta) / 4} .
$$

Proof Fix $f$ to be in the unit ball of $L^{(q / \theta)^{\prime}}\left(F^{n}, d x\right)$. It suffices to show that

$$
\|\hat{f}\|_{L^{p^{\prime}}(S, d \sigma)} \lesssim 1+R^{*}(p \rightarrow q)^{\theta}|F|^{-\tilde{d}(1-\theta) / 4} .
$$

Let $\lambda>0$ be a parameter to be chosen later. We can divide into two cases: either $|f(x)| \leq \lambda$ for all $x \in F^{n}$, or else $|f(x)| \geq \lambda$ for all $x$ in the support of $f$.

In the first case we have

$$
\|f\|_{L^{q^{\prime}}\left(F^{n}, d x\right)} \lesssim \lambda^{\left(q^{\prime}-(q / \theta)^{\prime}\right) / q^{\prime}}=\lambda^{-(1-\theta) /(q-\theta)}
$$

and so by (3) we have

$$
\|\hat{f}\|_{L^{p^{\prime}}(S, d \sigma)} \lesssim R^{*}(p \rightarrow q) \lambda^{-(1-\theta) /(q-\theta)} .
$$

Now suppose we are in the second case. We can estimate the $L^{p^{\prime}}(S, d \sigma)$ norm by the $L^{2}(S, d \sigma)$ norm and use Plancherel to obtain

$$
\|\hat{f}\|_{L^{p^{\prime}}(S, d \sigma)}^{2} \leq\left|\left\langle f, f *(d \sigma)^{\vee}\right\rangle\right|
$$

We split $(d \sigma)^{\vee}=\delta_{0}+K$. Using (15) we obtain

$$
\|\hat{f}\|_{L^{p^{\prime}(S, d \sigma)}}^{2} \leq\|f\|_{L^{2}\left(F^{n}, d x\right)}^{2}+|F|^{-\tilde{d} / 2}\|f\|_{L^{1}\left(F^{n}, d x\right)}^{2} .
$$

Since $d x$ is discrete we have

$$
\|f\|_{L^{2}\left(F^{n}, d x\right)} \leq\|f\|_{L^{(q / \theta)^{\prime}\left(F^{n}, d x\right)}} \leq 1
$$

while by the assumption $|f(x)| \geq \lambda$ on the support of $f$ we have

$$
\|f\|_{L^{1}\left(F^{n}, d x\right)} \lesssim \lambda^{1-(q / \theta)^{\prime}}=\lambda^{-\theta /(q-\theta)} .
$$

Thus we have

$$
\|\hat{f}\|_{L^{p^{\prime}}(S, d \sigma)} \lesssim 1+|F|^{-\tilde{d} / 2} \lambda^{-\theta /(q-\theta)} .
$$

To balance our two cases we define $\lambda>0$ by

$$
R^{*}(p \rightarrow q) \lambda^{-(1-\theta) /(q-\theta)}=|F|^{-\tilde{d} / 2} \lambda^{-\theta /(q-\theta)} .
$$

A little algebra then shows that (16) holds in both cases ${ }^{2}$.

\footnotetext{
${ }^{1}$ This idea of passing to a bilinear formulation and eliminating the diagonal case is quite useful in the Euclidean theory, see e.g. [42], [43], [44], [48].

${ }^{2}$ Alternatively, one can proceed by considering convolution with $\delta_{0}+|F|^{z} K$ where $z$ is a complex interpolation parameter; this is in the spirit of Stein's argument [36]. We omit the details.
} 
In particular, if we insert the bound $R^{*}(2 \rightarrow 2) \lesssim|F|^{(n-d) / 2}$ from (5) we obtain that $R^{*}(2 \rightarrow q)$ is bounded whenever

$$
q \geq 2+\frac{4(n-d)}{\tilde{d}} .
$$

In the Euclidean setting this bound on $q$ is often quite sharp (if $p$ is constrained to equal 2). This is usually due to the presence of "Knapp" examples for $S$, see e.g. [45]. The analogous type of example in the Euclidean case would be an arithmetic progression, or perhaps a subspace as in (11), but these are not always available.

To illustrate this let us again take the paraboloid (13). If $x=\left(x_{1}, \ldots, x_{n}\right)$ with $x_{n} \neq 0$, then

$$
\begin{aligned}
(d \sigma)^{\vee}(x) & =|F|^{1-n} \sum_{\xi \in F_{*}^{n-1}} e\left(x \cdot \xi+x_{n} \xi \cdot \xi\right) \\
& =|F|^{1-n} \prod_{i=1}^{n} \sum_{\xi_{i} \in F_{*}} e\left(x_{i} \xi_{i}+x_{n} \xi_{i} \xi_{i}\right) \\
& =|F|^{1-n} \prod_{i=1}^{n} e\left(x_{i} x_{i} / 4 x_{n}\right) \sum_{\xi_{i} \in F_{*}} e\left(x_{n}\left(\xi_{i}+x_{i} / 2 x_{n}\right)^{2}\right) \\
& =|F|^{1-n} S\left(x_{n}\right)^{n-1} e\left(\underline{x} \cdot \underline{x} / 4 x_{n}\right)
\end{aligned}
$$

where $\underline{x}:=\left(x_{1}, \ldots, x_{n-1}\right)$ and $S(x)$ is the Gauss sum

$$
S(x):=\sum_{\xi \in F_{*}} e\left(x \xi^{2}\right) .
$$

If $x_{n}=0$, then the above sum collapses instead to

$$
(d \sigma)^{\vee}(x)=\delta_{0}(\underline{x}) .
$$

As is well known, the magnitude of the Gauss sum is $|F|^{1 / 2}$. Indeed we have

$$
\begin{aligned}
|S(x)|^{2} & =\sum_{\xi, \eta \in F_{*}} e\left(x \xi^{2}\right) e\left(-x \eta^{2}\right) \\
& =\sum_{\xi, \eta \in F_{*}} e(x(\xi+\eta)(\xi-\eta)) \\
& =|F|+\sum_{\xi, \eta \in F_{*}: \xi \neq \eta} e(x(\xi+\eta)(\xi-\eta)) \\
& =|F|+\sum_{h \in F_{*}: h \neq 0} \sum_{\eta \in F} e(x(2 \eta+h) h) \\
& =|F|+\sum_{h \in F_{*}: h \neq 0} 0 .
\end{aligned}
$$

Thus (15) holds with $\tilde{d}:=d=n-1$. In particular we obtain that $R^{*}(2 \rightarrow q)$ is bounded whenever

$$
q \geq 2+\frac{4}{n-1}
$$


This does not compare well with the results of the previous section, although it does reproduce the result $R^{*}(2 \rightarrow 4)$ proved earlier in the case when $n=3$ and -1 is not a square. The condition (10) (or (9)) suggests that one should in fact obtain boundedness of $R^{*}(2 \rightarrow q)$ whenever

$$
q \geq 2+\frac{2}{n-1}
$$

which is indeed the case in two dimensions. However, in higher dimensions the paraboloid can contain subspaces in which case one can narrow the gap. For instance, when $n=3$ and $-1=i^{2}$ for some $i \in F$, then $S$ contains lines such as $\{(t, i t, 0): t \in F\}$ and so by (11) we obtain a necessary condition of $q \geq 4$, which does indeed match the Tomas-Stein exponent (19) in this case. However, this does not preclude that the bound of $R^{*}(2 \rightarrow 4)$ can be improved in other directions, indeed from (9), (11) one might conjecture that $R^{*}(3 \rightarrow 3)$ holds. (An analogous conjecture exists for the paraboloid in $\mathbf{R}^{3}$ ).

Similar examples can be made in higher odd dimensions (with $k:=(n-1) / 2)$, but there appears to be a gap between the Tomas-Stein exponent and the best known counterexamples when $n$ is even or when -1 is not a square. We shall not pursue these matters further, however we shall close this section with an improvement of the " $q$ " index of the Tomas-Stein restriction theorem $R^{*}(2 \rightarrow 4)$ in the case when $n=3$ and -1 is not a square.

Theorem 6.2. When $n=3, S$ is the paraboloid (13) and -1 is not a square, then $R^{*}(2 \rightarrow 18 / 5+\varepsilon) \leq C_{\varepsilon}$ for all $\varepsilon>0$.

Proof We shall exploit the restriction theorem in Proposition 5.2 as well as Fourier identities for the paraboloid which in the Euclidean setting have been exploited in [12]. We also use the close relationship between the restriction and Bochner-Riesz problems, first observed in [18].

By Lemma 6.1 with $p=2, q=16 / 5, \theta=8 / 9$ - it suffices to show that

$$
R^{*}(2 \rightarrow 16 / 5) \lesssim|F|^{1 / 16} \text {. }
$$

(Note from (8) that this is sharp up to logarithms). By (14) it suffices to show that

$$
\left|\left\langle f, g *(d \sigma)^{\vee}\right\rangle\right| \lesssim|F|^{1 / 8}\|f\|_{L^{16 / 11\left(F^{3}, d x\right)}}\|g\|_{L^{16 / 11}\left(F^{3}, d x\right)} ;
$$

for all $f, g$ on $F^{3}$. It suffices to show that

$$
\left|\left\langle f, g *(d \sigma)^{\vee}\right\rangle\right| \lesssim|F|^{1 / 8}\|f\|_{L^{4 / 3}\left(F^{3}, d x\right)}\|g\|_{L^{8 / 5}\left(F^{3}, d x\right)},
$$

since bilinear interpolation of this inequality with the one where $f$ and $g$ are exchanged gives the above estimate on $L^{16 / 11}$. By duality above inequality becomes

$$
\left\|g *(d \sigma)^{\vee}\right\|_{L^{4}\left(F^{3}, d x\right)} \lesssim|F|^{1 / 8}\|g\|_{L^{8 / 5}\left(F^{3}, d x\right)} .
$$

The $\delta_{0}$ portion of $(d \sigma)^{\vee}$ is definitely acceptable by Young's inequality, so it suffices to show

$$
\|g * K\|_{L^{4}\left(F^{3}, d x\right)} \lesssim|F|^{1 / 8}\|g\|_{L^{8 / 5}\left(F^{3}, d x\right)}
$$


where $K$ is the Bochner-Riesz kernel introduced earlier. We split $x:=\left(x_{1}, x_{2}, x_{3}\right)$, and for each $y_{3} \in F$ define the function $g_{y_{3}}$ to be the restriction of $g$ to the hyperplane $\left\{\left(x_{1}, x_{2}, x_{3}\right): x_{3}=y_{3}\right\}$. Clearly

$$
\sum_{y_{3} \in F}\left\|g_{y_{3}}\right\|_{L^{8 / 5}\left(F^{3}, d x\right)} \leq|F|^{3 / 8}\left(\sum_{y_{n} \in F}\left\|g_{y_{3}}\right\|_{L^{8 / 5}\left(F^{3}, d x\right)}^{8 / 5}\right)^{5 / 8}=|F|^{3 / 8}\|g\|_{L^{8 / 5}\left(F^{3}, d x\right)}
$$

so by the triangle inequality it suffices to show that

$$
\left\|g_{y_{3}} * K\right\|_{L^{4}\left(F^{3}, d x\right)} \lesssim|F|^{-1 / 4}\left\|g_{y_{3}}\right\|_{L^{8 / 5}\left(F^{3}, d x\right)}
$$

for each $y_{3}$. By translation invariance we may take $y_{3}=0$.

From (17) we have

$$
K\left(\underline{x}, x_{3}\right)=|F|^{-2} S\left(x_{3}\right)^{2} e\left(\underline{x} \cdot \underline{x} / 4 x_{3}\right)
$$

so we can write the left-hand side of $(20)$ as

$$
\left(\left.\left.\sum_{x_{3} \in F: x_{3} \neq 0} \sum_{\underline{x} \in F^{2}}|| F\right|^{-2}\left|S\left(x_{3}\right)\right|^{2} \sum_{\underline{y} \in F^{2}} g(\underline{y}, 0) e\left((\underline{x}-\underline{y}) \cdot(\underline{x}-\underline{y}) / 4 x_{3}\right)\right|^{4}\right)^{1 / 4} .
$$

By (18) we have $\left|S\left(x_{3}\right)\right|^{2}=|F|$ for $x_{3} \neq 0$. We now make the "pseudo-conformal" substitution $t:=1 / 4 x_{3}$ and $z:=-\underline{x} / 2 x_{3}$, so that

$$
(\underline{x}-\underline{y}) \cdot(\underline{x}-\underline{y}) / 4 x_{3}=z^{2} x_{3}+z \cdot \underline{y}+t \underline{y}^{2}
$$

and the previous expression becomes

$$
|F|^{-1}\left(\sum_{t \in F: t \neq 0} \sum_{z \in F^{2}}\left|e\left(z^{2} x_{3}\right) \sum_{\underline{y} \in F^{2}} g(\underline{y}, 0) e\left((z, t) \cdot\left(\underline{y}, \underline{y}^{2}\right)\right)\right|^{4}\right)^{1 / 4} .
$$

The phase $e\left(z^{2} x_{3}\right)$ can be discarded, as can the restriction $t \neq 0$. We can thus bound the previous by

$$
|F|^{-1}\left(\sum_{(z, t) \in F^{3}}\left|(G d \sigma)^{\vee}(z, t)\right|^{4}\right)^{1 / 4}
$$

where

$$
G\left(\underline{y}, \underline{y}^{2}\right):=|F|^{2} g(\underline{y}, 0) .
$$

By Proposition 5.2 we may bound this by

$$
\lesssim|F|^{-1}\|G\|_{L^{8 / 5}(S, d \sigma)}=|F|^{-1 / 4}\left\|g_{0}\right\|_{L^{8 / 5}\left(F^{3}, d x\right)},
$$

and the claim follows.

The logarithmic loss can probably be removed. Since the above restriction theorem uses one restriction estimate to prove another, it may be possible to iterate it (as in [42], [43]) to obtain some improvement, but this is unlikely to reach the conjectured best possible bound of $R^{*}(2 \rightarrow 3)$. This estimate also compares favorably with the best bound for the paraboloid in $\mathbf{R}^{3}$, which is $R^{*}(p \rightarrow 26 / 7+)$ for certain $2<p \leq \infty$, and it may be that the argument above also has some application to the Euclidean problem.

The question of what $L^{p} \rightarrow L^{q}$ estimates the convolution operator $f \mapsto f * K$ is itself quite interesting, being the finite field version of the Bochner-Riesz problem (see e.g. [6], [36] for a discussion). From the above argument we see that this problem 
is closely related to restriction, especially for paraboloids. (In the Euclidean case there are further connections, see [39], although the arguments there rely on scaling and so do not extend to the finite field case). We will not discuss this topic further here though.

\section{THE CONE}

We now consider the restriction problem for the cone

$$
S:=\left\{(\xi, u, v): \xi, u, v \in F_{*} ; u v=\xi^{2}\right\} \backslash\{(0,0,0)\}
$$

in $F^{3}$; the higher-dimensional cones are also of interest but will not be discussed here. We have removed the origin $(0,0,0)$ for technical convenience, but it can be restored with no significant change to the results.

It is relatively easy to obtain the boundedness of $R^{*}(2 \rightarrow 4)$ (the Euclidean counterpart of this is in [2], although the exponents are slightly different). One expects ${ }^{3}$ to apply Lemma 5.1, however there is a slight difficulty because the cone contains lines through the origin, which is bad for (12) when $\eta=0$. However, when $\eta \neq 0$ a routine algebraic computation shows that the number of solutions to (12) is $O(|F|)$ (in other words, the intersection of a cone with a non-trivial translate of itself is at most one-dimensional). By the argument in the proof of Lemma 5.1 we thus have

$$
\|f d \sigma * f d \sigma\|_{L^{2}\left(F_{*}^{3} \backslash\{0\}, d \xi\right)} \lesssim\|f\|_{L^{2}(S, d \sigma)}^{2} .
$$

On the other hand, a direct computation shows

$$
|f d \sigma * f d \sigma(0)| \lesssim|F|\|f\|_{L^{2}(S, d \sigma)}^{2},
$$

and the claim follows by summing.

Because the cone contains one-dimensional spaces (if we re-insert the origin) we obtain from (11) the necessary condition $q \geq 2 p^{\prime}$ for the boundedness of $R^{*}(p \rightarrow q)$. Thus the bound on $R^{*}(2 \rightarrow 4)$ is sharp in one sense. In fact it is the best restriction bound possible:

Proposition 7.1. If $q<4$ and $1 \leq p \leq \infty$, then $R^{*}(p \rightarrow q)$ is unbounded.

Proof The idea is to complete the square in the phase of the cone restriction operator and then use the standard estimate for Gauss sums.

We apply (3) with $f:=\chi_{X}$, where $X$ is the set

$$
X:=\left\{(x, y, z) \in F^{3}: z \text { is a non-zero square, and } y=x^{2} / 4 z\right\} .
$$

Clearly $|X| \sim|F|^{2}$. We will show that

$$
\left\|\hat{\chi}_{X}\right\|_{L^{p^{\prime}(S, d \sigma)}} \gtrsim|F|^{3 / 2},
$$

which implies the claim since $q>4$. In fact we will show that

$$
\left|\hat{\chi}_{X}(\xi, u, v)\right| \sim|F|^{3 / 2}
$$

\footnotetext{
${ }^{3}$ The decay here is $\tilde{d}=1$ and so a Tomas-Stein style argument would only yield the boundedness of $R^{*}(2 \rightarrow 6)$.
} 


\begin{tabular}{|l|l|l|}
\hline Surface & $\begin{array}{l}\text { Best known } \\
\text { restriction theorem }\end{array}$ & $\begin{array}{l}\text { Best known } \\
\text { counterexample }\end{array}$ \\
\hline$\left(t, \ldots, t^{n}\right)$, char $(F)>n$ & $R^{*}(2 \rightarrow 2 n)$ & $R^{*}(2 \rightarrow 2 n)$ \\
$n=2$, parabola & $R^{*}(2 \rightarrow 4)$ & $R^{*}(2 \rightarrow 4)$ \\
$n=3$, paraboloid, -1 non-square & $R^{*}(8 / 5 \rightarrow 4), R^{*}(2 \rightarrow 18 / 5+)$ & $R^{*}(2 \rightarrow 3)$ \\
$n=3$, paraboloid, -1 square & $R^{*}(2 \rightarrow 4)$ & $R^{*}(3 \rightarrow 3)$ \\
$n=3$, cone & $R^{*}(2 \rightarrow 4)$ & $R^{*}(2 \rightarrow 4)$ \\
\hline
\end{tabular}

Figure 1. Some of the surfaces discussed in this paper, the best restriction theorem we could prove (up to logarithms), and the conjectured best restriction theorem suggested by the counterexamples.

for all $(\xi, u, v) \in S$ with $u \neq 0$.

To see this, we write $v=\xi^{2} / u$ and $\xi=t u$, let $Q$ be the set of all non-zero squares in $F$, and compute

$$
\begin{aligned}
\hat{\chi}_{X}\left(t u, u, t^{2} u\right) & =\sum_{z \in Q} \sum_{x \in F} e\left(x t u+x^{2} u / 4 z+t^{2} u z\right) \\
& =\sum_{z \in Q} \sum_{x \in F} e\left(u(x+2 t z)^{2} / 4 z\right) \\
& =\sum_{z \in Q} \sum_{y \in F} e\left(u y^{2}\right) \quad(\text { substituting } y:=(x+2 t z) / \sqrt{2 z}) \\
& =|Q| S(u)
\end{aligned}
$$

and the claim follows from (18) and $2|Q|=|F|-1$.

Unlike the situation in Euclidean space $\mathbf{R}^{3}$, this counter-example does not seem to be removable simply by passing to a bilinear formulation. However, it may well be that the large body of work on the Euclidean cone restriction problem ([9], [31], [48], [43], etc.) still has some application to the finite field case.

We summarize our results on the restriction problem for finite fields in Figure 7 .

\section{The KaKeya PRoblem}

Having completed our discussion of restriction problems for the moment, we now set up the notation for the Kakeya problem.

We parameterize the vector space $F^{n}$ by $x=\left(\underline{x}, x_{n}\right)$, where $\underline{x} \in F^{n-1}, x_{n} \in F$.

For any $x_{0}, v \in F^{n-1}$, define the line $l\left(x_{0}, v\right)$ by

$$
l\left(x_{0}, v\right):=\left\{\left(x_{0}+v t, t\right): t \in F\right\} .
$$


We refer to $v$ as directions, and endow the space $F^{n-1}$ of directions with normalized counting measure

$$
\int_{F^{n-1}} f(v) d v:=\frac{1}{|F|^{n-1}} \sum_{v \in F^{n-1}} f(v) .
$$

In this definition we have excluded the horizontal lines, but this will make no essential difference to our results.

Define a Besicovitch set to be any subset $E$ of $F^{n}$ which contains a line in every direction, i.e. for every $v \in F^{n-1}$ there exists an $x_{0}$ such that $l\left(x_{0}, v\right) \in E$.

As an example of a Besicovitch set, and consider

$$
E:=\left\{(x, t) \in F^{2}: x+t^{2} \text { is a square }\right\} .
$$

This set has cardinality $\frac{1}{2}|F|^{2}+\frac{1}{2}|F|$, and for every $v \in F$ the line $l\left(v^{2} / 4, v\right)$ is contained in $E$. (cf. the "completing the square" trick in Proposition 7.1).

The Kakeya set conjecture for finite fields asserts that every Besicovitch set has cardinality $|E| \approx|F|^{n}$. Informally, this means that it is impossible to compress lines in distinct directions into a small set.

This conjecture is proven in two dimensions but is open in higher dimensions. In three dimensions the best bound is $|E| \gtrsim|F|^{5 / 2}$ (see [49] or the arguments below). For higher dimensions, see below.

There are many important variations of the Kakeya problem, in which lines are replaced by circles, planes, light rays, spheres, or other geometric objects, or if the requirement of distinct directions is replaced by some other condition. We do not attempt a survey of all the possibilities here, but refer the reader to [49].

One obvious attempt to construct a counterexample to the Kakeya set conjecture would be to set $E$ equal to some algebraic variety such as $\left\{x \in F^{n}: P(x)=0\right\}$ where $P: F^{n} \rightarrow F$ is some polynomial. However, this cannot work:

Proposition 8.1. Let $K>0$, and let $E$ be contained in the set

$$
\bigcup_{i=1}^{K}\left\{x \in F^{n}: P_{i}(x)=0\right\},
$$

where for each $1 \leq i \leq K, P_{i}: F^{n} \rightarrow F$ is a non-zero polynomial of degree at most $K$. Then, if char $(F)$ is sufficiently large depending on $K$, the set $E$ cannot be a Besicovitch set.

Proof Suppose for contradiction that $E$ is a Besicovitch set. Then by the pigeonhole principle for every $v \in F^{n-1}$, there exists $1 \leq i \leq K$ such that $l\left(x_{0}(v), v\right)$ intersects $\left\{x \in F^{n}: P_{i}(x)=0\right\}$ in at least $|F| / K$ points. By another pigeonholing, we may therefore find $1 \leq i \leq K$ and a set $V \subset F^{n-1}$ of cardinality $|V| \geq|F|^{n-1} / K$ such that

$$
\left|l\left(x_{0}(v), v\right) \cap\left\{x \in F^{n}: P_{i}(x)=0\right\}\right| \geq|F| / K
$$


for all $v \in V$. In other words,

$$
\left|\left\{t \in F: P_{i}\left(x_{0}(v)+t v, t\right)=0\right\}\right| \geq|F| / K \text { for all } v \in V .
$$

If $\operatorname{char}(F)$ is sufficiently large, this implies (since $P_{i}$ has bounded degree) that

$$
P_{i}\left(x_{0}(v)+t v, t\right)=0 \text { for all } v \in V \text { and } t \in F .
$$

Suppose that $P_{i}$ has degree $d$ for some $0<d \leq M$, and let $P_{i}^{*}$ be the principal part of $P_{i}$ (i.e. the terms which have degree exactly equal to $d$ ). Then $P_{i}\left(x_{0}(v)+t v, t\right)$ is a polynomial in $t$ of degree $d$. Extracting the $t^{d}$ component we see that

$$
P_{i}^{*}(t v, t)=0 \text { for all } v \in V \text { and } t \in F .
$$

Fix $t$, and think of $P_{i}^{*}(t v, t)$ as a polynomial in $v$ of degree at most $d$. This is zero on at least $1 / K$ of the values of $F^{n}$, which implies (if $\operatorname{char}(F)$ is sufficiently large) that $P_{i}^{*}(t v, t)$ is identically zero. But this implies that $P_{i}^{*}(x, t)=0$ for all $(x, t) \in F^{n}$, which is absurd since $P_{i}$ was supposed to have degree $d$. This is the desired contradiction.

Other attempts to produce counterexamples, e.g. by using the squares as in (23), or by random constructions, do not appear to give Besicovitch sets which are much smaller than $F^{n}$. However this is far from a proof that the Kakeya set conjecture is true.

The Kakeya set conjecture can be attacked directly, however we shall study it in the context of a more general conjecture concerning maximal functions.

For any function $f$ on $F^{n}$, define the Kakeya maximal function $f^{*}$ on $F^{n-1}$ by

$$
f^{*}(v)=\sup _{x_{0} \in F^{n-1}} \sum_{x \in l\left(x_{0}, v\right)}|f(x)| .
$$

Let $1 \leq p, q \leq \infty$ be exponents. We define $K(p \rightarrow q)$ to be the best constant such that

$$
\left\|f^{*}\right\|_{L^{q}\left(F^{n-1}, d v\right)} \leq K(p \rightarrow q)\|f\|_{L^{p}\left(F^{n}, d x\right)}
$$

for all $f$. By linearization and duality, this estimate is equivalent to the statement that

$$
\left\|\int_{v \in F^{n-1}} g(v) \chi_{l\left(x_{0}(v), v\right)} d v\right\|_{L^{p^{\prime}\left(F^{n}, d x\right)}} \leq K(p \rightarrow q)\|g\|_{L^{q^{\prime}\left(F^{n-1}, d v\right)}}
$$

for all $g$ on $F^{n-1}$, and all functions $x_{0}: F^{n-1} \rightarrow F^{n-1}$. Clearly we may take $f$ and $g$ non-negative in the above.

For instance, it is easy to verify that

$$
K(1 \rightarrow q)=1 \text { and } K(\infty \rightarrow q)=|F|
$$

for all $1 \leq q \leq \infty$, while from the Hölder and Young inequalities we see that $K(p \rightarrow q)$ is non-decreasing in both $p$ and $q$. By testing (25) with $f$ equal to the characteristic function of a point, line, or all of $F^{n}$, we obtain the bounds

$$
K(p \rightarrow q) \geq 1,|F|^{-(n-1) / q+1 / p^{\prime}},|F|^{1-n / p} .
$$


Thus in order for $K(p \rightarrow q)$ to be bounded one must have

$$
p \leq n \text { and } q \geq(n-1) p^{\prime} .
$$

The Kakeya maximal conjecture for finite fields asserts that these necessary conditions are also sufficient. In particular, $K(n \rightarrow n)$ should be bounded. The continuous version of this conjecture has been intensively studied; see [49] for a survey.

This conjecture is related to the Kakeya set conjecture in the following sense: if $K(p \rightarrow q)$ is bounded, then Besicovitch sets have cardinality at least $\gtrsim|F|^{p}$. To see this, observe from construction that if $E$ is a Besicovitch set, then

$$
\left(\chi_{E}\right)^{*}(v)=|F|
$$

for all directions $v$. Inserting this into (25) the claim follows.

Thus it is of interest to bound $K(p \rightarrow q)$ for as large a value of $p$ as possible. When $p=2$ this is quite easy:

Proposition 8.2 (Córdoba's argument). $K(2 \rightarrow 2 n-2) \leq \sqrt{2}$.

Proof We use (26), and compute

$$
\left\|\int_{v \in F^{n-1}} g(v) \chi_{l\left(x_{0}(v), v\right)} d v\right\|_{L^{2}\left(F^{n}, d x\right)}^{2}
$$

as

$$
\int_{v, v^{\prime} \in F^{n-1}} g(v) g\left(v^{\prime}\right)\left|l\left(x_{0}(v), v\right) \cap l\left(x_{0}\left(v^{\prime}\right), v\right)\right| d v d v^{\prime} .
$$

First consider the diagonal contribution $v=v^{\prime}$. This is

$$
\frac{1}{|F|^{n-1}} \int_{F^{n-1}} g(v)^{2}|F| d v=|F|^{2-n}\|g\|_{L^{2}\left(F^{n-1}, d v\right)}^{2} \leq\|g\|_{L^{(2 n-2)^{\prime}\left(F^{n-1}, d v\right)}}^{2} .
$$

Now consider the off-diagonal contribution $v \neq v^{\prime}$. Since two non-parallel lines can only intersect in at most one point, this contribution is at most

$$
\int_{v, v^{\prime} \in F^{n-1}} g(v) g\left(v^{\prime}\right) d v d v^{\prime}=\|g\|_{L^{1}\left(F^{n-1}, d v\right)}^{2} \leq\|g\|_{L^{(2 n-2)^{\prime}\left(F^{n-1}, d v\right)}}^{2} .
$$

Adding the two terms, the claim follows.

Observe that this estimate is on the line $q=(n-1) p^{\prime}$, and implies that Besicovitch sets have cardinality at least $\frac{1}{2}|F|^{2}$ (compare with the example (23)). This settles the Kakeya conjectures in two dimensions.

In the remainder of our discussion we shall be prepared to lose logarithmic factors. In this case the Kakeya problem is equivalent to the classic problem of counting incidences between points and lines, but with the condition that the lines all point in different directions.

Proposition 8.3. Let $1 \leq p, q \leq \infty$. The statement $K(p \rightarrow q) \lesssim 1$ holds if and only if one has the incidence bound

$$
|\{(p, l) \in P \times L: p \in l\}| \lesssim|P|^{1 / p}|L|^{1 / q^{\prime}}|F|^{(n-1) / q}
$$


for all collections $P$ of points in $F^{n}$ and all collections $L$ of lines in $F^{n}$, each of which points in a different direction.

Proof Suppose first that $K(p \rightarrow q) \lesssim 1$, and let $P$ and $L$ be as above. Let $V \subset F^{n-1}$ denote the directions of the lines in $L$, then we see that

$$
|\{(p, l) \in P \times L: p \in l\}| \leq \sum_{v \in V}\left(\chi_{P}\right)^{*}(v)=|F|^{n-1} \int_{V}\left(\chi_{P}\right)^{*} .
$$

Applying Hölder and (25) and noting that $|V|=|L|$, the claim (27) follows.

Now suppose that (27) holds. By (26) and duality it suffices to show that

$$
\sum_{x \in F^{n}} \int_{v \in F^{n-1}} g(v) \chi_{l\left(x_{0}(v), v\right)}(x) f(x) d v \lesssim\|g\|_{L^{q^{\prime}\left(F^{n-1}, d v\right)}}\|f\|_{L^{p}\left(F^{n}, d x\right)} .
$$

By the standard dyadic pigeonholing argument we may assume that $f=\chi_{P}$ and $g=\chi_{V}$ are characteristic functions. (Note that since there are at most $|F|^{n}$ points and directions, we only need to pigeonhole into $O(\log |F|)$ categories). The above estimate then becomes

$$
|F|^{1-n} \sum_{x \in P} \sum_{v \in V} \chi_{l\left(x_{0}(v), v\right)}(x) \lesssim|F|^{(1-n) / q^{\prime}}|V|^{1 / q^{\prime}}|P|^{1 / p} .
$$

But this follows from (27) by setting $L:=\left\{l\left(x_{0}(v), v\right): v \in V\right\}$.

As discussed before we have the constraint $q \geq(n-1) p^{\prime}$ as a necessary condition for (27) to hold. However if one is prepared to lose the trivial error term of $|P|+|L|$ then one can do much better; see Proposition 5.3, or Proposition 8.6 below. To prepare for this we give

Corollary 8.4. Suppose that we can prove a bound of the form

$$
|\{(p, l) \in P \times L: p \in l\}| \lesssim|P|^{a}|L|^{1-b}|F|^{1-c}+|P|+|L|
$$

for some $0 \leq a, b, c \leq 1$ with $(n-1) b+c \geq 1$, whenever $L$ has distinct directions. Then we have

$$
K(p \rightarrow q) \lesssim 1
$$

where $p:=\frac{1}{a}((n-1) b+c)$ and $\left.q:=\min \left((n-1) p^{\prime}, \frac{1}{b}(n-1) b+c\right)\right)$.

Proof Denote the left-hand side of (28) by $|I|$. By (27) it suffices to prove

$$
\frac{|I|}{|L||F|} \lesssim|P|^{1 / p}\left(\frac{|L|}{|F|^{n-1}}\right)^{1 / q}|F|^{-1}
$$

If $|I| \lesssim|P|$ then (29) follows from the trivial bounds $|P| \leq|F|^{n}$ and $|L| \geq 1$ since $q \geq(n-1) p^{\prime}$. If $|I| \lesssim|L|$ then (29) follows from the trivial bounds $|L| /|F|^{n-1} \leq 1$ and $|P| \geq 1$. By hypothesis we may therefore assume

$$
\frac{|I|}{|L||F|} \lesssim|P|^{a}\left(\frac{|L|}{|F|^{n-1}}\right)^{-b}|F|^{-(n-1) b-c} \text {. }
$$

The claim then follows by interpolating this with the trivial bound $|I| \leq|L||F|$ (since every line contains at most $|F|$ points). 
Thus, for instance, Proposition 5.3 can be used to imply Proposition 8.2 in the two dimensional case (at least if one is willing to lose logarithms). It is an interesting question to ask whether Proposition 5.3 can be improved. Consider for instance the case $|L|=|P|$, in which case the Proposition gives an incidence bound of $|P|^{3 / 2}$. This is sharp when $|P|=|F|^{2}$ (just set $P$ and $L$ equal to the space of all points and all lines), and more generally for any subfield $G$ of $F$ the estimate is sharp when $|P|=|G|^{2}$ (set $P$ equal to $G^{2}$, and $L$ equal to lines with slope and intercept in $G)$. However, one expects to do better when $|F|$ does not contain any sub-fields. For instance, if $F=\mathbf{Z} / p \mathbf{Z}$ for some prime $p$ and $|P|=|L|=p$, is it possible to substantially improve the incidence bound of $p^{3 / 2}$ ? In the Euclidean case one would obtain a bound of $O\left(p^{4 / 3}\right)$ from the famous Szemerédi-Trotter theorem [38], but this argument uses crucially the fact that $\mathbf{R}$ is an ordered field and so does not apply to $\mathbf{Z} / p \mathbf{Z}$.

It turns out that the question of improving the bound of $p^{3 / 2}$ is equivalent to disproving the existence of a set $E \subset \mathbf{Z} / p \mathbf{Z}$ for which $|E| \approx|E+E| \approx|E \cdot E| \approx \sqrt{p}$. See [24], [41], [17]. In other words, the question is equivalent to whether $\mathbf{Z} / p \mathbf{Z}$ contains an "approximate sub-ring" of "dimension $1 / 2$ ".

There are similar connections between the continuous versions of these problems, specifically the Falconer distance problem, the Furstenburg set problem, and the Erdös ring problem. See [24], [41].

The argument in Proposition 5.3 extends without difficulty to higher dimensions ${ }^{4}$, and one can thus conclude using Proposition 8.3 that $K((n+1) / 2 \rightarrow(n+1)) \lesssim 1$. This is the analogue of the results in [15] (see also [16]), and shows that Besicovitch sets have cardinality $\gtrsim|F|^{(n+1) / 2}$.

We now improve this to $(n+2) / 2$ by the following argument of Wolff ([46], [49]; we shall use an argument due to Nets Katz).

Definition 8.5. A collection $L$ of lines in $F^{n}$ obeys the Wolff axiom if every 2-plane contains at most $O(|F|)$ lines from $L$.

Clearly any collection $L$ of lines with distinct directions will obey the Wolff axiom.

Proposition 8.6. If $L$ obeys the Wolff axiom, then

$$
|\{(p, l) \in P \times L: p \in l\}| \lesssim|P|^{1 / 2}|L|^{3 / 4}|F|^{1 / 4}+|P|+|L| .
$$

Proof Denote the set on the left-hand side of (30) by $I$. We may assume without loss of generality that we have the "two-ends condition"

$$
|I| \gg|P|
$$

(i.e. most lines contain at least two points) and the "bilinear condition"

$$
|I| \gg|L|
$$

\footnotetext{
${ }^{4}$ Of course, one loses duality of points and lines in higher dimensions, however the only geometric fact we needed was that every two distinct lines are incident to at most one point, and this fact is preserved on the dual side (i.e. two distinct points determine at most one line).
} 
(i.e. most points lie in at least two lines) since (30) is trivial otherwise.

The idea will be to obtain a lower and upper bound for the number of triangles in $F^{n}$ formed by $P$ and $L$.

Let $L^{\prime}$ denote the set of lines with the average or above-average number of points in $P$ :

$$
L^{\prime}:=\{l \in L:|l \cap P| \geq|I| / 2|L|\} .
$$

Let $I^{\prime}:=\left\{(p, l) \in I: l \in L^{\prime}\right\}$. Clearly we have $\left|I \backslash I^{\prime}\right|<|I| / 2$, so that

$$
\left|I^{\prime}\right| \sim|I| \text {. }
$$

Let $V$ denote the set of (possibly degenerate) "angles"

$$
V:=\left\{\left(p, l, l^{\prime}\right) \in P \times L^{\prime} \times L^{\prime}:(p, l),\left(p, l^{\prime}\right) \in I^{\prime}\right\} .
$$

From (1) we have

$$
|V| \geq \frac{\left|I^{\prime}\right|^{2}}{|P|} \sim \frac{|I|^{2}}{|P|}
$$

Let $V^{\prime}$ denote the non-degenerate angles $V^{\prime}:=\left\{\left(p, l, l^{\prime}\right) \in V: l \neq l^{\prime}\right\}$. Clearly $\left|V \backslash V^{\prime}\right|=|I|$. By (31) and the preceding we thus have

$$
\left|V^{\prime}\right| \gtrsim \frac{|I|^{2}}{|P|} \text {. }
$$

Let $W$ denote the set of non-degenerate pointed angles

$$
W=\left\{\left(p, l, l^{\prime}, p^{\prime}\right) \in V^{\prime} \times P: p^{\prime} \in l^{\prime}, p \neq p^{\prime}\right\} .
$$

From the definition of $L^{\prime}$, each element of $V^{\prime}$ contributes at least $\frac{|I|}{2|L|}-1$ elements to $W$. From (32) we thus have

$$
|W| \gtrsim \frac{|I|\left|V^{\prime}\right|}{|L|} .
$$

Let $T$ denote the space of pairs of linked non-degenerate pointed angles:

$$
T=\left\{\left(p_{1}, l_{1}, l_{1}^{\prime}, p_{1}^{\prime}, p_{2}, l_{2}, l_{2}^{\prime}, p_{2}^{\prime}\right) \in W \times W: l_{1}=l_{2}, p_{1}^{\prime}=p_{2}^{\prime}\right\} .
$$

The set $T$ can be identified with the space of triangles with points and lines in $P$ and $L$, although there is a possible degeneracy in that $p_{1}$ may equal $p_{2}$. From (35) and (1) we thus have

$$
|T| \geq \frac{|W|^{2}}{|P||L|} .
$$

Now let $T^{\prime}$ be the space of non-degenerate triangles:

$$
T^{\prime}=\left\{\left(p_{1}, l_{1}, l_{1}^{\prime}, p_{1}^{\prime}, p_{2}, l_{2}, l_{2}^{\prime}, p_{2}^{\prime}\right) \in T: p_{1} \neq p_{2}\right\} .
$$

The set $T \backslash T^{\prime}$ is bijective with $W$. From the previous estimates and (31) we thus have $\left|T \backslash T^{\prime}\right|=|W| \ll|T|$, thus

$$
\left|T^{\prime}\right| \gtrsim \frac{|W|^{2}}{|P||L|} .
$$

This is our lower bound for the number of triangles. To obtain an upper bound, we observe from the Wolff axiom that $p_{1}, l_{1}, l_{1}^{\prime}$ contributes at most $|F|$ elements to $T^{\prime}$. This is because the line $l_{2}^{\prime}$ lies in the plane generated by $l_{1}, l_{1}^{\prime}$. The remaining 
variables are determined by $\left\{p_{1}^{\prime}\right\}=\left\{p_{2}^{\prime}\right\}=l_{1}^{\prime} \cap l_{2}^{\prime},\left\{p_{2}\right\}=l_{2} \cap l_{2}^{\prime}$, and $l_{2}=l_{1}$. Since $\left(p_{1}, l_{1}, l_{1}^{\prime}\right) \in V^{\prime}$, we thus have

$$
\left|T^{\prime}\right| \leq\left|V^{\prime}\right||F|
$$

Combining the previous two estimates with (35) we obtain

$$
\left|V^{\prime}\right||F| \gtrsim \frac{|I|^{2}\left|V^{\prime}\right|^{2}}{|P||L|^{3}}
$$

Dividing by $\left|V^{\prime}\right|$ and then applying (34) we obtain

$$
|F| \gtrsim \frac{|I|^{4}}{|P|^{2}|L|^{3}}
$$

and (30) follows.

From the above and Corollary 8.4 we thus have ${ }^{5}$ that

$$
K\left(\frac{n+2}{2} \rightarrow \frac{(n-1)(n+2)}{n}\right) \lesssim 1
$$

This is the analogue of the main result of [46], and also lies on the line $q=(n-1) p^{\prime}$. In particular, Besicovitch sets have cardinality $\gtrsim|F|^{(n+2) / 2}$. In fact one can remove the logarithm by appealing directly to Proposition 8.6.

In the important three-dimensional case we do not know if this bound of $(n+2) / 2=$ $5 / 2$ can be improved. However, we cannot improve this bound solely by using the Wolff axiom and the cardinality bound on $L$. To see this, suppose that $F$ has a subfield $G$ of index 2 , and let $P$ be the Heisenberg group

$$
P:=\left\{\left(z_{1}, z_{2}, z_{3}\right) \in F: \operatorname{Im}\left(z_{1} \overline{z_{2}}\right)=\operatorname{Im}\left(z_{3}\right)\right\}
$$

where $z \mapsto \bar{z}$ is the non-identity involution which preserves $G$, and $\operatorname{Im}(z):=(z-$ $\bar{z}) / 2$. The set $P$ has cardinality $\sim|F|^{5 / 2}$, and contains the family of lines

$$
L:=\left\{l\left(\left(x_{1}, x_{2}\right),\left(v_{1}, v_{2}\right)\right): \operatorname{Im}\left(v_{1} \overline{v_{2}}\right)=\operatorname{Im}\left(x_{1} \overline{x_{2}}\right)=0 ; v_{1} \overline{x_{2}}+v_{2} \overline{x_{1}}=1\right\} .
$$

This family of lines can be seen to have cardinality $\sim|F|^{2}$ and obeys the Wolff axiom, but the lines do not all point in distinct directions. Thus to improve the $(n+2) / 2$ bound in three dimensions one must somehow use the distinctness of the directions more intimately ${ }^{6}$. On the other hand, it may be possible to improve $(n+2) / 2$ in higher dimensions just by using the Wolff axiom (together with the obvious generalizations to subspaces of dimension greater than 2).

One possible approach to the Kakeya problem which does indeed exploit distinctness of directions is via arithmetic combinatorics (see [10], [23], [25]). We borrow the following notation from [25].

\footnotetext{
${ }^{5}$ It is clear from the proof of Corollary 8.4 that (30) in fact is substantially stronger than this Kakeya estimate, especially with the two-ends and bilinear assumptions. This is consistent with experience in the Euclidean case, see e.g. [46] for a discussion of the two-ends property, and [42] for the bilinear property.

${ }^{6}$ Alternatively, if one is working in a field such as $F=\mathbf{Z} / p \mathbf{Z}$, one could try to preclude approximate half-dimensional rings of the type mentioned earlier, in order to eliminate Heisenbergtype counterexamples.
} 
Define a slope $r$ to be any element of

$$
\left\{\frac{a}{b}: a, b \in \mathbf{Z}: 0<b<\operatorname{char}(F)\right\} \cup\{\infty\} .
$$

We define the projections $\pi_{r}: F^{n-1} \times F^{n-1} \rightarrow F^{n-1}$ by $\pi_{r}(a, b):=a+r b$ for $r \neq \infty$ and $\pi_{\infty}(a, b):=b$. We call a slope proper if $r \neq-1$.

Definition 8.7. Let $R$ be a finite collection of proper slopes, and let $\alpha \in \mathbf{R}$. We say that the statement $S D(R, \alpha)$ holds if one has the bound $|G| \lesssim \sup _{r \in R}\left|\pi_{r}(G)\right|^{\alpha}$ whenever $G \subseteq F^{n-1} \times F^{n-1}$ is a finite set obeying

$$
\pi_{-1} \text { is one-to-one on } G \text {. }
$$

One can think of the statement $S D(R, \alpha)$ as a quantitative way to control the cardinality of the projection $\{a-b:(a, b) \in G\}$ in terms of other projections $\{a+r b:(a, b) \in G\}$.

One trivially has $S D(R, 2)$ as soon as $R$ contains at least two elements. In [10] Bourgain improved this to $S D\left(\{0,1, \infty\}, 2-\frac{1}{13}\right)$ based on some arguments of Gowers [20]; this was later improved to $S D\left(\{0,1, \infty\}, 2-\frac{1}{6}\right)$ in [23], or $S D\left(\{0,1,2, \infty\}, 2-\frac{1}{4}\right)$ if $\operatorname{char}(F)$ is sufficiently large. The current record is that for any $F$ there exists a finite set $R$ and an $\varepsilon_{F}>0$ such that $\varepsilon_{F} \rightarrow 0$ as $\operatorname{char}(F) \rightarrow \infty$ and $S D\left(R, \alpha+\varepsilon_{F}\right)$ holds, where $\alpha=1.675 \ldots$ is the largest root of $\alpha^{3}-4 \alpha+2=0$ (see [25]; the statement there is for vector spaces but the extension to finite fields of sufficiently large characteristic is routine).

These problems are related to the Balog-Szemeredi theorem [1] (see also [20]). Their connection to Kakeya lies through relationships such as the following.

Lemma 8.8. If $S D(R, \alpha)$ holds for some $R$, then Besicovitch sets have cardinality $\gtrsim|F|^{(n-1) / \alpha+1}$ (the implicit constant depends on $R$ ).

In particular, if one could prove sums-differences lemmas with $\alpha$ arbitrarily close to one, one would be able to settle the Kakeya set conjecture. To improve upon the three-dimensional results one would need $\alpha$ to be better than $4 / 3$.

Proof We follow the arguments of Bourgain [10]. Let $E$ be a Besicovitch set, and let $C_{0}$ be a large number (depending on $R$ ) to be chosen later. Call a height $t \in F$ exceptional if

$$
\left|\left\{x \in F^{n-1}:(x, t) \in E\right\}\right| \geq C_{0}|E| /|F| .
$$

Clearly at most $|F| / C_{0}$ heights are exceptional. By a simple probabilistic argument we thus see that if $C_{0}$ is sufficiently large, then we may find distinct heights $t_{0}, t_{\infty} \in$ $F$ such that the heights

$$
t_{r}:=\frac{1}{r+1} t_{0}+\frac{r}{r+1} t_{\infty}
$$

are non-exceptional for all $r \in R$.

Fix $t_{0}, t_{\infty}$, and define

$$
G:=\left\{\left(x_{0}(v)+t_{0} v, x_{0}(v)+t_{\infty} v\right): v \in F^{n-1}\right\} .
$$


Since $t_{0} \neq t_{\infty}$, we see that $\pi_{-1}$ is one-to-one on $G$ and

$$
|G|=|F|^{n-1} \text {. }
$$

On the other hand, since $\left(x_{0}(v)+t_{r} v, t_{r}\right) \in E$ for all $v \in F^{n-1}$ and $r \in R$, we have

$$
\left|\pi_{r}(G)\right| \leq\left|\left\{x \in F^{n-1}:\left(x, t_{r}\right) \in E\right\}\right| \leq C_{0}|E| /|F| .
$$

Applying $S D(R, \alpha)$ the claim follows.

This Lemma immediately gives some bounds on the size of Besicovitch sets, for instance the result $S D\left(\{0,1, \infty\}, 2-\frac{1}{4}\right)$ gives $|E| \gtrsim|F|^{(4 n+3) / 7}$ for sufficiently large values of $\operatorname{char}(F)$. We give a direct proof of this fact below:

Proposition 8.9. [23] If $\operatorname{char}(F)>3$, then we have $|E| \gtrsim|F|^{(4 n+3) / 7}$.

Proof We set $P$ equal to $E$, and $L$ equal to the lines $L=\left\{l\left(x_{0}(v), v\right): v \in F^{n-1}\right\}$. Assume for contradiction that $|P| \ll|F|^{(4 n+3) / 7}$.

Let $I=\{(p, l) \in P \times L: p \in L\}$ be the set of incidences, then $|I|=|F|^{n}$. Let $V^{\prime}$ denote the non-degenerate angles

$$
V^{\prime}:=\left\{\left(p, l_{1}, l_{2}\right) \in P \times L \times L: p \in l_{1} ; p \in l_{2} ; l_{1} \neq l_{2}\right\} .
$$

Arguing as in Proposition 8.6 we have

$$
\left|V^{\prime}\right| \gtrsim \frac{|I|^{2}}{|P|}=|F|^{2 n}|P|^{-1}
$$

Thus if we let $A$ denote the set

$$
A:=\left\{\left(p, p_{1}, p_{2}, l_{1}, l_{2}\right) \in P^{3} \times L^{2}: p, p_{1} \in l_{1} ; p, p_{2} \in l_{2} ; l_{1} \neq l_{2} ; p \neq p_{1} ; p \neq p_{2}\right\}
$$

we have

$$
|A| \gtrsim|F|^{2}\left|V^{\prime}\right| \gtrsim|F|^{2 n+2}|P|^{-1}
$$

The pair $\left(p_{1}, p_{2}\right)$ lies in a set of cardinality $|P|^{2}$, so by (1) we have

$$
|Q| \gtrsim \frac{|A|^{2}}{|P|^{2}} \gtrsim|F|^{4 n+4}|P|^{-4}
$$

where $Q$ denotes the set of quadrilaterals

$$
\begin{aligned}
Q:=\{ & \left(p, p^{\prime}, p_{1}, p_{2}, l_{1}, l_{2}, l_{1}^{\prime}, l_{2}^{\prime}\right) \in P^{4} \times L^{4}: \\
& p, p_{1} \in l_{1} ; p, p_{2} \in l_{2} ; p^{\prime}, p_{1} \in l_{1}^{\prime} ; p^{\prime}, p_{2} \in l_{2}^{\prime} ; \\
& \left.p, p^{\prime} \neq p_{1}, p_{2} ; l_{1} \neq l_{2} ; l_{1}^{\prime} \neq l_{2}^{\prime}\right\} .
\end{aligned}
$$

We can pass to the non-degenerate quadrilaterals

$$
Q^{\prime}:=\left\{\left(p, p^{\prime}, p_{1}, p_{2}, l_{1}, l_{2}, l_{1}^{\prime}, l_{2}^{\prime}\right) \in Q: p \neq p^{\prime}\right\}
$$

since $p=p^{\prime}$ forces $\left(p, p_{1}, p_{2}, l_{1}, l_{2}\right)=\left(p^{\prime}, p_{1}, p_{2}, l_{1}^{\prime}, l_{2}^{\prime}\right)$, and so the cardinality of $Q \backslash Q^{\prime}$ is at most $|A| \ll|A|^{2} /|P|^{2} \lesssim|Q|$. Thus

$$
\left|Q^{\prime}\right| \gtrsim|F|^{4 n+4}|P|^{-4}
$$

Now consider the map $f: Q^{\prime} \rightarrow P^{3}$ defined by

$$
f\left(p, p^{\prime}, p_{1}, p_{2}, l_{1}, l_{2}, l_{1}^{\prime}, l_{2}^{\prime}\right):=\left(\frac{1}{2} p+\frac{1}{2} p_{1},-p_{1}+2 p^{\prime}, \frac{2}{3} p^{\prime}+\frac{1}{3} p_{2}\right) .
$$


This triple lies in $l_{1} \times l_{1}^{\prime} \times l_{2}^{\prime}$ and is thus in $P^{3}$ as claimed. Now we consider to what extent an element $q \in Q$ is determined by $f(q)$.

Write $q=\left(p, p^{\prime}, p_{1}, p_{2}, l_{1}, l_{2}, l_{1}^{\prime}, l_{2}^{\prime}\right)$, and suppose that $f(q)$ is fixed. From the identity

$$
p-p_{2}=2\left(\frac{1}{2}+\frac{1}{2} p_{1}\right)+\left(-p_{1}+2 p^{\prime}\right)-3\left(\frac{2}{3} p^{\prime}+\frac{1}{3} p_{2}\right)
$$

we see that $p-p_{2}$ is also fixed. But $p, p_{2} \in l_{2}$, so $p-p_{2}$ is parallel to the direction of $l_{2}$. Since the lines in $L$ all point in different directions, we thus see that $l_{2}$ is fixed. This gives at most $|F|$ choices for $p$ (say). Once $p$ is chosen, the remaining components of $q$ are determined since $f(q)$ is fixed (the lines $l_{1}, l_{2}, l_{1}^{\prime}, l_{2}^{\prime}$ are then uniquely determined by the distinct points $\left.p, p_{1}, p_{2}, p^{\prime}\right)$.

To summarize, for each fixed value of $f(q)$ there are at most $|F|$ values of $q$. Since there are at most $|P|^{3}$ values of $f(q)$, we thus have

$$
\left|Q^{\prime}\right| \lesssim|P|^{3}|F|
$$

Combining this with (38) we obtain $|P| \lesssim|F|^{(4 n+3) / 7}$ as desired.

This argument can be combined with Wolff's argument in Proposition 8.6 to obtain further improvements, from $(4 n+3) / 7$ to $(4 n+5) / 7$ (or better) if $\operatorname{char}(F)$ is sufficiently large. See [25]. Some of these arguments also extend to the maximal function problem, giving estimates of a type similar to (30). We will not pursue these matters here, but remark that anyone interested in improving the known Kakeya results in the Euclidean case might first consider the finite field case in which several technical difficulties (e.g. small angles and small separations) are no longer present ${ }^{7}$.

\section{The Connection between Restriction and KaKeya}

Until now we have pursued the restriction and Kakeya problems independently, with only a very slight connection between the two (via Proposition 5.3). In the Euclidean case there is a much stronger relationship between the two problems. For instance, when $S$ is the paraboloid

$$
S:=\left\{\left(\xi,|\xi|^{2}\right): \xi \in \mathbf{R}^{n-1} ;|\xi| \lesssim 1\right\}
$$

one can use a Taylor approximation at scale $R^{-1 / 2}$ for some $R \gg 1$ to approximate the paraboloid to within $O(1 / R)$ by the union of flat disks

$$
\bigcup_{\xi \in R^{-1 / 2} \mathbf{Z}^{n-1}:|\xi| \lesssim 1}\left(\xi,|\xi|^{2}\right)+\left\{(\eta, 2 \xi \cdot \eta):|\eta| \lesssim R^{-1 / 2}\right\} .
$$

A function supported on one of these disks would have Fourier transform concentrated along tubes pointing in the normal direction. Since all the disks point in different directions, we thus begin to see Kakeya-type objects appearing. For more precise discussions we refer to [6], [8], [3], [32], [42], [43].

\footnotetext{
${ }^{7}$ On the other hand certain Euclidean arguments ([22], [28]) require an induction on scales type argument which is not directly reproducible for finite fields (unless perhaps they have subfields of index 2).
} 
To obtain the analogous results for finite fields we cannot work just with the standard paraboloid as there is no Taylor approximation in finite fields. Instead, we force the Taylor approximation into existence by fiat, by defining the surface

$$
\tilde{S}:=\left\{(\xi, \xi \cdot \xi, \eta, \xi \cdot \eta): \eta, \theta \in F^{n-1}\right\} \subset F^{2 n}
$$

(we have dropped the factor of 2 as it makes no difference).

The restriction theory for $S$ and $\tilde{S}$ are related to each other, and to the Kakeya problem, by the following theorem.

Theorem 9.1. Let $2 \leq p, q$ be exponents. Then we have

$$
\begin{gathered}
R_{S}^{*}(p \rightarrow q) \leq R_{\tilde{S}}^{*}(p \rightarrow q), \\
K\left((q / 2)^{\prime} \rightarrow(p / 2)^{\prime}\right) \leq R_{\tilde{S}}^{*}(p \rightarrow q)^{2}|F|^{n-1-\frac{2 n}{p}}
\end{gathered}
$$

and

$$
R_{\tilde{S}}^{*}(p \rightarrow q) \leq R_{S}^{*}(2 \rightarrow q) K\left((q / 2)^{\prime} \rightarrow(p / 2)^{\prime}\right)^{1 / 2}
$$

The estimate (40) shows how restriction estimates imply Kakeya estimates, and is the finite field analogue of the result in [3]. At the endpoint $p=2 n /(n-1)$ the result is especially interesting given that there is no loss of powers of $|F|$ in this case. The estimates (41), (39) give a partial converse and is the analogue of the arguments in [6], [8].

Proof We first prove (39). Let $f$ be any function on $F^{n}$. If we define the function $\tilde{f}$ on $F^{2 n}$ by

$$
\tilde{f}(x, y):=f(x) \delta_{y, 0}
$$

for all $x, y \in F^{n}$, we see that

$$
\widehat{\tilde{f}}(\eta, \eta \cdot \eta, \theta, \eta \cdot \theta)=\widehat{f}(\eta, \eta \cdot \eta)
$$

and hence that

$$
\|\widehat{\tilde{f}}\|_{L^{q}(\tilde{S}, d \sigma)}=\|\widehat{f}\|_{L^{q}(S, d \sigma)} .
$$

Since $\|\tilde{f}\|_{L^{p}\left(F^{2 n}, d x d y\right)}=\|f\|_{L^{p}\left(F^{n}, d x\right)}$, the claim (39) then follows from (3).

Now we prove (40). By (26) it suffices to show that

$\left\|\frac{1}{|F|^{n-1}} \sum_{v \in F^{n-1}} h(v) \chi_{l\left(x_{0}(v), v\right)}\right\|_{L^{p / 2}\left(F^{n}, d x\right)} \leq R_{\tilde{S}}^{*}(p \rightarrow q)^{2}|F|^{n-1-\frac{2 n}{p}}\|h\|_{L^{q / 2}\left(F^{n-1}, d v\right)}$

for all functions $h$ on $F^{n-1}$ and choices of map $x_{0}: F^{n-1} \rightarrow F^{n-1}$.

Fix $h, x_{0}$; we may assume that $h$ is non-negative. Define the function $\tilde{h}$ on $\tilde{S}$ by

$$
\tilde{h}(\eta, \eta \cdot \eta, \theta, \eta \cdot \theta)=h(-\eta)^{1 / 2} e\left(-x_{0}(-\eta) \cdot \theta\right) .
$$

Clearly

$$
\|\tilde{h}\|_{L^{q}(\tilde{S}, d \sigma)}=\left\|h^{1 / 2}\right\|_{L^{q}\left(F^{n-1}, d v\right)}=\|h\|_{L^{q / 2}\left(F^{n-1}, d v\right)} .
$$

Applying (3) we thus obtain

$$
\left\|(\tilde{h} d \sigma)^{\vee}\right\|_{L^{p}\left(F^{2 n}, d x\right)} \leq R_{\tilde{S}}^{*}(p \rightarrow q)\|h\|_{L^{q / 2}(S, d \sigma)}^{1 / 2} .
$$


For $(\tilde{h} d \sigma)^{\vee}\left(\underline{x}, x_{n}, \underline{y}, y_{n}\right)$ we get

$\frac{1}{|F|^{2(n-1)}} \sum_{\eta \in F^{n-1}} \sum_{\theta \in F^{n-1}} e\left(\underline{x} \cdot \eta+x_{n} \eta \cdot \eta+\underline{y} \cdot \theta+y_{n} \eta \cdot \theta\right) e\left(-x_{0}(-\eta) \cdot \theta\right) h(-\eta)^{1 / 2}$.

Performing the $\theta$ summation we get

$$
\frac{1}{|F|^{n-1}} \sum_{\eta \in F^{n-1}} e\left(\underline{x} \cdot \eta+x_{n} \eta \cdot \eta\right) h(-\eta)^{1 / 2} \delta_{\underline{y}, x_{0}(-\eta)-y_{n} \eta} .
$$

Writing $x=\left(\underline{x}, x_{n}\right), y=\left(\underline{y}, y_{n}\right)$, this becomes

$$
(\tilde{h} d \sigma)^{\vee}(x, y)=\frac{1}{|F|^{n-1}} \sum_{\eta \in F^{n-1}} e(x \cdot(\eta, \eta \cdot \eta)) h(-\eta)^{1 / 2} \chi_{l\left(x_{0}(-\eta),-\eta\right)}(y) .
$$

Making the change of variables $v=-\eta$, we thus have

$$
\left\|\sum_{v \in F^{n-1}} e(x \cdot(-v, v \cdot v)) h(v)^{1 / 2} \chi_{l\left(x_{0}(v), v\right)}(y)\right\|_{l_{y}^{p} l_{x}^{p}} \leq R_{\tilde{S}}^{*}(p \rightarrow q)|F|^{n-1}\|h\|_{L^{q / 2}(S, d \sigma)}^{1 / 2} .
$$

Since $p \geq 2$, we have from Hölder's inequality that

$$
\|f\|_{l_{x}^{2}} \leq|F|^{n\left(\frac{1}{2}-\frac{1}{p}\right)}\|f\|_{l_{x}^{p}}
$$

and hence

$\left\|\sum_{v \in F^{n-1}} e(x \cdot(-v, v \cdot v)) h(v)^{1 / 2} \chi_{l\left(x_{0}(v), v\right)}(y)\right\|_{l_{y}^{p} l_{x}^{2}} \leq R_{\tilde{S}}^{*}(p \rightarrow q)|F|^{\frac{n}{2}+\frac{n}{p^{\prime}}-1}\|h\|_{L^{q / 2}(S, d \sigma)}^{1 / 2}$.

By orthogonality we have

$\left\|\sum_{v \in F^{n-1}} e(x \cdot(-v, v \cdot v)) h(v)^{1 / 2} \chi_{l\left(x_{0}(v), v\right)}(y)\right\|_{l_{x}^{2}}=|F|^{n / 2}\left(\sum_{v \in F^{n-1}} h(v) \chi_{l\left(x_{0}(v), v\right)}(y)\right)^{1 / 2}$.

Thus we have

$$
\left\|\left(\sum_{v \in F^{n-1}} h(v) \chi_{l\left(x_{0}(v), v\right)}(y)\right)^{1 / 2}\right\|_{l_{y}^{p}} \leq R_{\tilde{S}}^{*}(p \rightarrow q)|F|^{\frac{n}{p^{r}}-1}\|h\|_{L^{q / 2}(S, d \sigma)}^{1 / 2} .
$$

Squaring this and then dividing by $|F|^{n-1}$, we obtain the desired estimate (40).

Finally, we prove (41), i.e.

$$
\left\|(h d \sigma)^{\vee}\right\|_{L^{p}\left(F^{2 n}, d x d y\right)}^{2} \leq R_{S}^{*}(2 \rightarrow q)^{2} K\left((q / 2)^{\prime} \rightarrow(p / 2)^{\prime}\right)\|h\|_{L^{q}(\tilde{S}, d \sigma)}^{2}
$$

for all $h$ on $\tilde{S}$.

We split $h=\sum_{\alpha \in F^{n-1}} h_{\alpha}$, where $h_{\alpha}$ is the restriction of $h$ to the "cap"

$$
C_{\alpha}=\left\{(\alpha, \alpha \cdot \alpha, \theta, \alpha \cdot \theta): \theta \in F^{n-1}\right\} .
$$

Fix $y=\left(\underline{y}, y_{n}\right)$ and expand

$$
\left(h_{\alpha} d \sigma\right)^{\vee}(x, y)=\frac{1}{|F|^{2(n-1)}} \sum_{\theta \in F^{n-1}} e\left(x \cdot(\alpha, \alpha \cdot \alpha)+\left(\underline{y}+\alpha y_{n}\right) \cdot \theta\right) \quad h(\alpha, \alpha \cdot \alpha, \theta, \alpha \cdot \theta) .
$$


We shall write this simply as

$$
\left(h_{\alpha} d \sigma\right)^{\vee}(x, y)=\frac{1}{|F|^{n-1}} e(x \cdot(\alpha, \alpha \cdot \alpha)) H\left(\alpha, \underline{y}+y_{n} \alpha\right)
$$

where $H(\alpha, z)$ is a function which depends on $h$ and $\alpha, z \in F^{n-1}$, but not on $x$.

Hence by using (2) we get

$$
\begin{aligned}
\left\|(h d \sigma)^{\vee}\right\|_{L^{q}\left(F^{2 n}, d x d y\right)}^{2} & =\left\|\frac{1}{|F|^{n-1}} \sum_{\alpha \in F^{n-1}} e(x \cdot(\alpha, \alpha \cdot \alpha)) H\left(\alpha, \underline{y}+y_{n} \alpha\right)\right\|_{l_{y}^{q} l_{x}^{q}}^{2} \\
& \leq R_{S}^{*}(2 \rightarrow q)^{2}\left\|\frac{1}{|F|^{n-1}} \sum_{\alpha \in F^{n-1}}\left|H\left(\alpha, \underline{y}+y_{n} \alpha\right)\right|^{2}\right\|_{l_{y}^{q / 2}} .
\end{aligned}
$$

For a suitable function $g \in l_{y}^{(q / 2)^{\prime}}$ with norm 1 we may express the latter squared $l_{y}^{q / 2}$-norm as

$$
\frac{1}{|F|^{n-1}} \sum_{\underline{y} \in F^{n-1}} \sum_{y_{n} \in F} \sum_{\alpha \in F^{n-1}} g\left(\underline{y}, y_{n}\right)\left|H\left(\alpha, \underline{y}+y_{n} \alpha\right)\right|^{2} .
$$

By replacing $\underline{y}$ by $\underline{y}-y_{n} \alpha$ and summing over $y_{n}$ first according to (24) we may estimate this by

$$
\frac{1}{|F|^{n-1}} \sum_{\alpha \in F^{n-1}} g^{*}(-\alpha) \sum_{\underline{y} \in F^{n-1}}|H(\alpha, \underline{y})|^{2}
$$

which by Plancherel is equal to

$$
\frac{1}{|F|^{n-1}} \sum_{\alpha \in F^{n-1}} g^{*}(-\alpha) \frac{1}{|F|^{n-1}} \sum_{\theta \in F^{n-1}}|h(\alpha, \alpha \cdot \alpha, \theta, \alpha \cdot \theta)|^{2} .
$$

By Hölder's inequality and the Kakeya estimate (25) we may bound this by

$$
K\left((q / 2)^{\prime} \rightarrow(p / 2)^{\prime}\right)\left(\frac{1}{|F|^{n-1}} \sum_{\alpha \in F^{n-1}}\left(\frac{1}{|F|^{n-1}} \sum_{\theta \in F^{n-1}}|h(\alpha, \alpha \cdot \alpha, \theta, \alpha \cdot \theta)|^{2}\right)^{p / 2}\right)^{2 / p} .
$$

Since $p \geq 2$ we can bound the inner $l_{\theta}^{2}$-norm by Hölder's inequality and obtain (41) by collecting terms.

As a sample application of these estimates, we consider the $n=3$ case in which -1 is not a square. Interpolating between (36) and Proposition 8.2 we have

$$
K(9 / 4 \rightarrow 18 / 5) \lesssim 1
$$

Combining this with Theorem 6.2 and (41) we have

$$
R_{\tilde{S}}^{*}(36 / 13 \rightarrow 18 / 5) \lesssim 1
$$

which by Lemma 6.1 implies (since the Fourier transform of $d \sigma$ on $\tilde{S}$ can easily be computed to have some decay)

$$
R_{\tilde{S}}^{*}(36 / 13 \rightarrow 18 / 5+\varepsilon) \leq C_{\varepsilon}
$$

for all $\varepsilon>0$.

Let us now consider the necessary conditions for $R_{\tilde{S}}^{*}(p \rightarrow q)$ to be bounded. From (39) the conditions must be at least as restrictive as those for $S$, in particular we 
must have $q \geq 2 n /(n-1)$ from (9). The condition (10) gives $q \geq n p^{\prime} /(n-1)$, but this can be improved to $q \geq(n+1) p^{\prime} /(n-1)$ by applying (11) with $(n, d, k)$ replaced by $(2 n, 2(n-1), n-1)$. (Note that this latter condition is almost obeyed with equality by (44)). In analogy with the Euclidean restriction conjecture for the paraboloid (which has almost the same numerology, except that one must have strict inequality in $q>2 n /(n-1)$ because of the multiplicity of scales) one may tentatively conjecture that these necessary conditions are also sufficient (at least when -1 is not a square). From (40) this conjecture would imply the Kakeya set conjecture. The relevant endpoint is $R_{S^{*}}^{*}(2 n /(n-1) \rightarrow 2 n /(n-1))$; one can show this endpoint is bounded in two dimensions by direct computation but the problem remains open in higher dimensions. In three dimensions we see from (41) that this would follow from the endpoint restriction bound $R_{S}^{*}(2 \rightarrow 3)$ for the ordinary paraboloid, combined with the endpoint Kakeya bound $K(3 \rightarrow 3)$.

\section{REFERENCES}

[1] A. Balog, E. Szemerédi, A statistical theorem of set addition, Combinatorica, 14 (1994), 263-268.

[2] B. Barcelo, On the restriction of the Fourier transform to a conical surface, Trans. Amer. Math. Soc. 292 (1985), 321-333.

[3] W. Beckner, A. Carbery, S. Semmes, F. Soria, A note on restriction of the Fourier transform to spheres., Bull. London Math. Soc. 21 (1989), no. 4, 394-398.

[4] B. Bollobas, Extremal Graph Theory, Academic Press, London 1978.

[5] J. Bourgain, Bounded orthogonal systems and the $\Lambda(p)$-set problem. Acta Math. 162 (1989), $227-245$.

[6] J. Bourgain, Besicovitch-type maximal operators and applications to Fourier analysis, Geom. and Funct. Anal. 22 (1991), 147-187.

[7] J. Bourgain, Fourier restriction phenomena for certain lattice subsets and applications to nonlinear evolution equations, Part I, Geometric and Funct. Anal. 3 (1993), 107-156.

[8] J. Bourgain, Some new estimates on oscillatory integrals, Essays in Fourier Analysis in honor of E. M. Stein, Princeton University Press (1995), 83-112.

[9] J. Bourgain, Estimates for cone multipliers, Operator Theory: Advances and Applications, 77 (1995), 41-60.

[10] J. Bourgain, On the dimension of Kakeya sets and related maximal inequalities, Geom. Funct. Anal. 9 (1999), no. 2, 256-282.

[11] J. Bourgain, Harmonic analysis and combinatorics: How much may they contribute to each other?, Mathematics: Frontiers and perspectives, IMU/Amer. Math. Society 2000, 13-32.

[12] A. Carbery, Restriction implies Bochner-Riesz for paraboloids, Math. Proc. Cambridge Philos. Soc. 111 (1992), no. 3, 525-529.

[13] L. Carleson and P. Sjölin, Oscillatory integrals and a multiplier problem for the disc, Studia Math. 44 (1972): 287-299.

[14] M. Christ, Restriction of the Fourier transform to submanifolds of low codimension, thesis, U. of Chicago, 1982.

[15] M. Christ, J. Duoandikoetxea, and J. L. Rubio de Francia, Maximal operators associated to the Radon transform and the Calderón-Zygmund method of rotations, Duke Math. J. 53 (1986), 189-209.

[16] S. Drury, $L^{p}$ estimates for the x-ray transform, Ill. J. Math. 27 (1983), 125-129.

[17] G. Elekes, On the number of sums and products, Acta Arith. 81 (1997), 365-367.

[18] C. Fefferman, Inequalities for strongly singular convolution operators, Acta Math. 124 (1970), 9-36.

[19] J.E. Fournier, Majorants and $L^{p}$ norms, Israel J. Math, 18 (1974), 157-166.

[20] T. Gowers, A new proof of Szemerédi's theorem for arithmetic progressions of length four, to appear in GAFA. 
[21] G.H. Hardy and J.E. Littlewood. Notes on the theory of series (XIX): a problem concerning majorants of Fourier series., Quart. J. Math., 6 (1935), 304-315.

[22] N. Katz, I. Laba, T. Tao, An improved bound for the Minkowski dimension of Besicovitch sets in $\mathbf{R}^{3}$, Annals of Math. 152 (2000), 383-446.

[23] N. Katz, T. Tao, Bounds on arithmetic projections, and applications to the Kakeya conjecture, Math Res. Letters 6 (1999), 1-6.

[24] N. Katz, T. Tao, Some connections between the Falconer and Furstenburg conjectures, submitted, New York J. Math.

[25] N. Katz, T. Tao, New bounds for Kakeya problems, submitted, Journal d'Analyse de Jerusalem.

[26] J.E. Littlewood. Some Problems in Real and Complex Analysis. D.C.Heath, New York, 1968.

[27] S. Klainerman, M. Machedon, Space-time Estimates for Null Forms and the Local Existence Theorem, Comm. Pure Appl. Math., 46 (1993), 1221-1268.

[28] I. Łaba, T. Tao: An improved bound for the Minkowski dimension of Besicovitch sets in medium dimension, to appear.

[29] G. Mockenhaupt, Bounds in Lebesgue spaces of Oscillatory Integral Operators, Habilitationsschrift, Siegen 1996.

[30] G. Mockenhaupt, A restriction theorem for the Fourier transform, Bull. Amer. Math. Soc. 25 (1991), 31-36.

[31] G. Mockenhaupt, A note on the cone multiplier, Proc. AMS 117 (1993), 145-152.

[32] A. Moyua, A. Vargas, L. Vega, Schrödinger Maximal Function and Restriction Properties of the Fourier transform, International Math. Research Notices 16 (1996).

[33] I. Ruzsa, Sums of finite sets, Number Theory: New York Seminar; Springer-Verlag (1996), D.V. Chudnovsky, G.V. Chudnovsky and M.B. Nathanson editors.

[34] P. Sjölin, Regularity of solutions to Schrödinger equations, Duke Math. J.,55 (1987), 699-715.

[35] E. M. Stein, Some problems in harmonic analysis, Harmonic analysis in Euclidean spaces (Proc. Sympos. Pure Math., Williams Coll., Williamstown, Mass., 1978), Part 1, pp. 3-20.

[36] E. M. Stein, Harmonic Analysis, Princeton University Press, 1993.

[37] R. S. Strichartz, Restriction of Fourier Transform to Quadratic Surfaces and Decay of Solutions of Wave Equations, Duke Math. J., 44 (1977), 70 5-774.

[38] E. Szemerédi, W. T. Trotter Jr., Extremal problems in discrete geometry, Combinatorica 3 (1983), 381-392.

[39] T. Tao, The Bochner-Riesz conjecture implies the Restriction conjecture, Duke Math J. 96 (1999), 363-376.

[40] T. Tao, Multilinear weighted convolution of $L^{2}$ functions, and applications to non-linear dispersive equations, Amer. J. Math. 123 (2001), 839-908.

[41] T. Tao, Finite field analogues of the Erdos, Falconer, and Furstenburg problems, unpublished.

[42] T. Tao, A. Vargas, L. Vega, A bilinear approach to the restriction and Kakeya conjectures, J. Amer. Math. Soc. 11 (1998), pp. 967-1000.

[43] T. Tao, A. Vargas, A bilinear approach to cone multipliers I. Restriction Estimates, GAFA 10 (2000), 185-215.

[44] T. Tao, A. Vargas, A bilinear approach to cone multipliers II. Applications, GAFA 10 (2000), 216-258.

[45] P. Tomas, A restriction theorem for the Fourier transform, Bull. Amer. Math. Soc. 81 (1975), $477-478$.

[46] T. H. Wolff, An improved bound for Kakeya type maximal functions, Revista Mat. Iberoamericana. 11 (1995). 651-674.

[47] T. H. Wolff, A mixed norm estimate for the x-ray transform, Revista Mat. Iberoamericana. 14 (1998), 561-600.

[48] T. H. Wolff, A sharp bilinear cone restriction estimate, Annals of Math. 153(3) (2001), 661-698.

[49] T. Wolff, Recent work connected with the Kakeya problem, Prospects in mathematics (Princeton, NJ, 1996), 129-162, Amer. Math. Soc., Providence, RI, 1999.

[50] A. Zygmund, Trigonometric Series, Cambridge University Press, 1990. 
Georgia Institute of Technology Atlanta, GA 30332-0160

E-mail address: gerdm@math.gatech.edu

Department of Mathematics, UCLA, Los Angeles CA 90095-1555

E-mail address: tao@math.ucla.edu 